\title{
Differential Space-Time Shift Keying-Aided Successive-Relaying-Assisted Decode-and-Forward Cooperative Multiuser CDMA
}

\author{
Peichang Zhang, Sheng Chen, Fellow, IEEE, and Lajos Hanzo, Fellow, IEEE
}

\begin{abstract}
A differential space-time shift keying (DSTSK)aided successive-relaying-assisted multiuser decode-and-forward (DF) cooperative system is proposed. We employ low-complexity noncoherent detection, which requires channel state information (CSI) at neither the relay nodes (RNs) nor at the destination node (DN). More explicitly, the source nodes (SNs) employ differentially encoded phase-shift keying (PSK) modulation, whereas the RNs perform soft-input-soft-output multiple-symbol differential sphere decoding (SISO-MSDSD)-based DF relaying during the relaying phase. Similarly, DSTSK transmission is employed at the RNs, which is detected with the aid of SISO-MSDSD at the DN. More explicitly, three-stage serial-concatenated turbo encoding/decoding is employed throughout the system to enhance the attainable performance. Additionally, a maximum-minimum determinant-based configuration selection (MMBCS) algorithm is proposed to select the optimal DSTSK configuration for supporting a specific number of users. Moreover, we adopt a successiverelaying architecture for recovering the conventional $50 \%$ half-duplex relaying-induced throughput loss at the cost of supporting less users.
\end{abstract}

Index Terms-Differential space-time shift keying (DSTSK), multiple-symbol differential sphere decoding (MSDSD), multiusers, successive-relaying cooperation, virtual antenna array (VAA).

\section{INTRODUCTION}

\section{A. MIMO Overview}

I N RECENT years, mobile communication techniques having an increased reliability and bandwidth efficiency have been conceived. multiple-input-multiple-output (MIMO) techniques have attracted substantial attention due to their capability of providing both spatial diversity and multiplexing gains [1], [2]. As a more recent concept of MIMO systems, spatial modulation (SM) [3], [4] and space shift keying (SSK) [5] were proposed, where the basic idea is to activate only a single one of the $M$ antenna elements (AEs) during the transmission of each symbol, leading to a novel technique of conveying

Manuscript received October 19, 2011; revised October 14, 2012; accepted December 23, 2012. Date of publication January 23, 2013; date of current version June 12,2013 . This work was supported by RC-UK under the auspicies of the India-UK Advanced Technology Centre, by the European Union under the Concerto project, as well as by the European Research Council's Advanced Fellow Grant. The review of this paper was coordinated by Prof. M. Uysal.

The authors are with Electronics and Computer Science, University of Southampton, SO17 1BJ Southampton, U.K. (e-mail: pz3g09@ecs.soton.ac.uk; sqc@ecs.soton.ac.uk; 1h@ecs.soton.ac.uk).

Digital Object Identifier 10.1109/TVT.2013.2239673 source information. Furthermore, the interantenna Interference (IAI) is eliminated, and no interantenna synchronization is required. Therefore, low-complexity single-antenna-based maximum-likelihood (ML) detection becomes feasible. However, since SM/SSK was designed for achieving a high multiplexing gain, rather than a diversity gain, combating the effects of fading channels has to rely on the employment of multiple AEs at the receiver, which becomes extremely challenging in downlink scenarios owing to the limited size of shirt-pocket mobile devices. Additionally, the total number of transmit antennas required by SM/SSK to convey $m$ information bits is $2^{m}$, implying that the number of transmit antennas increases exponentially for a linear increase in the transmission rate [6].

Inspired by the concepts of SM/SSK, space-time shift keying (STSK) was proposed in [6], which is shown to have the following advantages over conventional SM/SSK schemes.

1) In the STSK scheme, one of the $Q$ appropriately indexed space-time dispersion matrices is activated within each STSK signal block duration, rather than activating a specific antenna at each symbol duration, which was the case in SM/SSK schemes. As a result, STSK beneficially exploits both the spatial and time dimensions.

2) Due to the high degree of design freedom, a flexible diversity versus a multiplexing gain tradeoff can be struck by optimizing both the number and size of the dispersion matrices, as well as the number of receive antennas. Moreover, according to [6], STSK schemes are capable of achieving both transmit and receive diversity gains, which eliminates the lack of transmit diversity gain experienced by conventional SM/SSK schemes, where only receive diversity gains can be attained.

Similar to SM/SSK systems, the IAI of the STSK system is also eliminated, and consequently, the adoption of low-complexity single-antenna-based ML detection becomes realistic.

\section{B. Cooperative Systems Overview}

Since achieving MIMO-aided transmit diversity in the mobile uplink becomes impractical due to the limited size of mobile handsets, as a more recent concept, cooperative communication was proposed for allowing the nodes to assist each other by forwarding messages to the destination [7], either in amplify-and-forward (AF) or decode-and-forward (DF) mode [8]. In [9], dual-hop relaying was proposed for SM systems, which was shown to result in an improved end-to-end system 
performance. An AF relaying cooperative system was proposed for SSK systems in [10] for achieving a transmit diversity gain by forming a virtual antenna array (VAA) [11], where several single-antenna-aided RNs cooperatively share their antennas. As a benefit, their random locations will result in mutually uncorrelated Rayleigh fading. In conventional two-phase cooperative communication systems [12]-[15], a multiplexing loss of 50\% is encountered due to the half-duplex transmitand-receive constraint of practical transceivers. In [16] and [17], an information-guided relaying system was proposed for increasing the throughput of the half-duplex relaying systems. Additionally, the concept of successive relaying was proposed in [18] for recovering the half-duplex multiplexing loss.

The performance of coherent MIMO systems relying on realistic imperfect channel state information (CSI) was analyzed in [19]-[22] to quantify the system's performance degradation, owing to imperfect CSI. Cooperative communication systems employing coherent detectors require accurate CSI at the receivers. Since channel estimation (CE) techniques [23], [24] exploit the fact that the consecutive time-domain samples of each of the channel impulse response (CIR) taps are correlated, obeying a correlation, which is commensurate with the velocity of the vehicle, both the pilot symbol overhead and the CE complexity increase, as the vehicular speed increases. This implies having more rapidly fluctuating CIR taps. Additionally, in cooperative communication systems, as the number of source nodes (SNs) and relay nodes (RNs) increases, the acquisition of accurate CSI for the increasing number of mobile-to-mobile channels becomes unrealistic while imposing excessive CE complexity [13]. In contrast to classic coherent detectors, the family of differentially encoded noncoherent detectors requires no CSI at the receivers; hence they constitute an attractive design alternative [25], [26]. Furthermore, since noncoherent receivers usually suffer from the well-known SNR penalty of $3 \mathrm{~dB}$, the multiple-symbol differential decoding (MSDD) algorithm [27] can be applied for mitigating the performance degradation, albeit at the cost of exponentially increased complexity upon extending the MSDD detection window size. The concept of multiple-symbol differential sphere decoding (MSDSD) was proposed by Lampe et al. [28] for reducing the detection complexity, while enhancing the attainable bit-error-ratio (BER) performance. As a further advance, the soft-input-soft-output MSDSD (SISO-MSDSD) is capable of achieving substantial iteration gains [29].

Direct-sequence code-division multiple-access (DS-CDMA) schemes are capable of achieving high capacity and flexibility; hence, they have found favor in both the second- and third-generation wireless communication systems [30], [31]. Additionally, multicarrier CDMA (MC-CDMA) is capable of achieving a potentially better performance than orthogonal frequency-division multiplexing (OFDM) because, if a few chips of a spreading code are corrupted, MC-CDMA may still be capable of recovering the related subcarrier signal. By contrast, in OFDM systems, the subcarrier's symbol is irreversibly corrupted if the subcarrier is attenuated by frequency-domain fading. Hence, it is anticipated that the future evolution of high-speed packet access and Long Term Evolution is likely to witness the return to MC-CDMA transceivers [30], [32], [33].

\section{Our Novel Contributions}

Against this background, the novel contribution of this paper is that we first propose a DSTSK-aided multiuser successiverelaying cooperative system (MUSRC). By exploiting the flexibility of the concept of DSTSK, our system is capable of supporting different numbers of users by appropriately adjusting the constellation size of the phase-shift keying (PSK) modulation scheme employed by DSTSK. Then, we opted for using binary PSK (BPSK), quadrature PSK (QPSK), 8-PSK, etc., conceived with a variable number of dispersion matrices. Additionally, our system is capable of activating a different number of relays by adjusting the dimensions of each dispersion matrix. We also proposed a novel maximum-minimum determinantbased configuration selection (MMBCS) algorithm activating the most appropriate DSTSK configuration in support of a specific number of users. Furthermore, since we adopted a SISOMSDSD-aided noncoherent detector in the proposed multiuser cooperative system, the system's complexity is significantly reduced, whereas a good BER performance is attained as a benefit of the powerful three-stage serial-concatenated turbo encoding/ decoding regime employed. Since we apply the successiverelaying philosophy of [18] in our system, the 50\% throughput loss of conventional two-phase relaying is recovered at the cost of supporting less users. Finally, the DS-CDMA system is adopted throughout our system to suppress the multiple-access interference.

The rest of this paper is organized as follows. The proposed DSTSK-aided MUSRC system is detailed in Section II, whereas the proposed MMBCS algorithm is presented in Section III. The system achievable performance is investigated in Section IV. Our conclusions are presented in Section V.

The following notational conventions are adopted in our discussions. A DSTSK system employing an $\mathcal{L}$-PSK modulation scheme is denoted as $\operatorname{DSTSK}\left(N_{r}, N, T, Q, \mathcal{L}\right)$, where $N_{r}$ indicates the number of relays in each VAA and $N$ is the number of receive antennas at the DN, whereas $T$ denotes the number of time slots occupied by the signal block and $Q$ is the number of dispersion matrices employed. The transpose and conjugate transpose operators are represented by $(\cdot)^{T}$ and $(\cdot)^{H}$, respectively, whereas $\|\cdot\|$ and $|\cdot|$ denote the norm and magnitude operators, respectively. The Kronecker product is denoted by $\otimes$, the conjugate operator by $(\cdot)^{*}$, and the $(M \times M)$ identity matrix by $\mathbf{I}_{M}$. Finally, $\operatorname{diag}\{\mathbf{s}\}$ is the diagonal matrix having the elements of $\mathbf{s}$ as its diagonal elements, $\varepsilon\{\cdot\}$ is the expectation operator, $\operatorname{tr}[\cdot]$ is referred to as the trace operator, and $\operatorname{det}[\cdot]$ is the determinant operator.

\section{Differential Space-Time Shift Keying-Aided MultiUSER SUCCESSIVE-RELAYING COOPERATIVE SYSTEM}

The block diagram of the proposed DSTSK-aided MUSRC system is depicted in Fig. 1, where the two-phase relaying network consists of $K$ SNs (users), $2 N_{r}$ RNs, and a destination node (DN). Due to the limited size of the shirt-pocket mobile devices, the SNs and RNs are all limited to have a single antenna. By contrast, the number of AEs at the DN depends on the configuration of the DSTSK scheme adopted. DS-CDMA is 


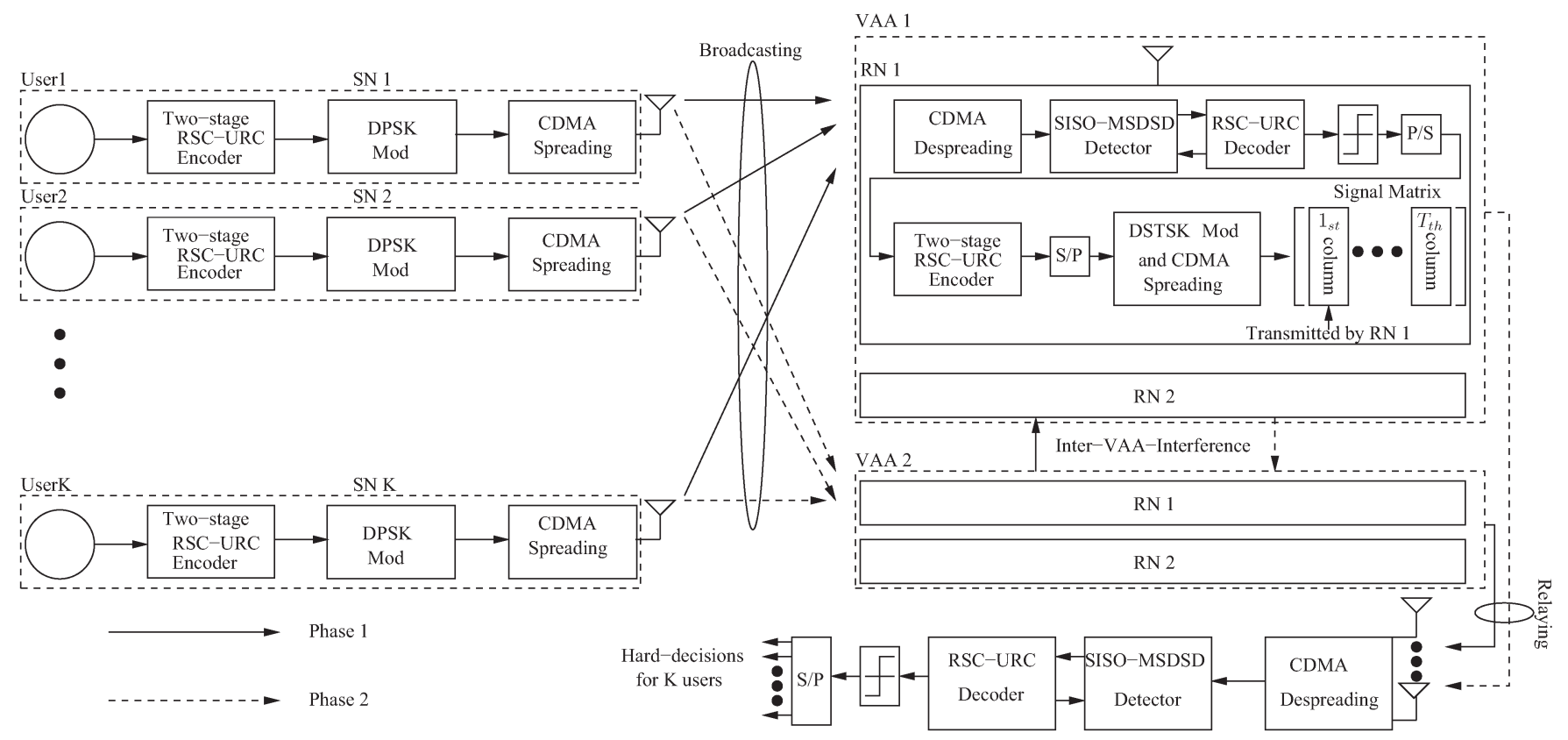

Fig. 1. Block diagram of the DSTSK-aided MUSRC that implements the two-phase successive relaying with the aid of two VAAs. In this example, the number of relays in each VAA is $N_{r}=2$, implying that the number of time slots is $T=2$.

adopted as the multiple-access technique [30]. Since two-phase successive relaying is employed, the number of VAAs in the network is $N_{\mathrm{VAA}}=2$. The number of RNs in each of the two VAA groups is $N_{r}=T$ since the number of transmit antennas should be equal to the number of time slots for DSTSK for the sake of achieving the maximum attainable transmit diversity gain [34].

\section{A. System Overview}

The operation of our MUSRC system is based on a two-phase (Phase 1 and Phase 2) alternating principle, where each operating phase contains two concurrent transmissions referred to as broadcasting and relaying transmissions, which are outlined as follows.

1) Phase 1: The operation of Phase 1 is represented in Fig. 1 by the solid-line arrows. During the broadcast phase, the information bits of each of the $K \mathrm{SNs}$ are first channel encoded by the two-stage serial-concatenated recursivesystematic-code-unity-rate-code (RSC-URC) encoder in Fig. 2 at the SNs. The channel-coded bits are mapped to the differential PSK (DPSK) constellation symbols for generating $K$ differentially encoded symbol sequences, which are spread by the $K$ user-specific spreading codes. The CDMA-spread signals are transmitted from each of the $K \mathrm{SNs}$ to the first VAA (VAA1), which is formed by $N_{r}$ RNs. At the RNs of VAA1, the signals received from the SNs are first despread, and then decoded by the three-stage serial-concatenated decoder in Fig. 2, generating the $K$ decoded signals of the $K$ users.

During the relaying transmission, the decoded users' signals, which are acquired from the most recent broadcast slot, are encoded by the two-stage RSC-URC encoder of Fig. 2 at VAA2, which are further modulated by the DSTSK modulator before being spread by the VAA-specific spreading codes, and finally transmitted to the DN. At the DN, the signal received from VAA2 is despread and then decoded by the three-stage serial-

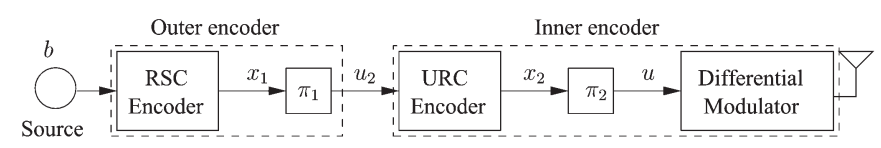

(a)

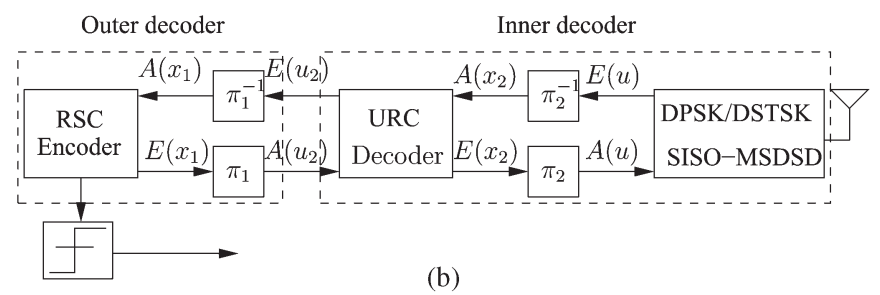

Fig. 2. Structure of three-stage serial-concatenated turbo encoder/decoder. (a) Three-stage turbo encoder. (b) Three-stage turbo decoder.

concatenated decoder of Fig. 2. Finally, the hard decisions are generated for the $K$ users to complete Phase 1.

2) Phase 2: The operations of Phase 2 are depicted in Fig. 1 by the dashed-line arrows, which are exactly identical to those in Phase 1, except for the roles of VAA1 and VAA2, which are swapped. More explicitly, in Phase 2, VAA2 is in the broadcast mode, whereas VAA1 is in the relaying transmission mode. This way, the successive relaying becomes capable of recovering the conventional 50\% throughput loss.

To suppress the successive-relaying-induced inter-VAA interference (IVI), the classic DS-CDMA technique is adopted at the RNs, where each VAA is assigned a specific spreading code, which implies that $N_{\mathrm{VAA}}$ spreading codes will be adopted by the VAAs in the network. As a result, the number of users supported is reduced by $N_{\mathrm{VAA}}$, albeit in reality, only the users roaming at the cell edge will rely on relaying.

\section{B. Three-Stage Turbo Encoder/Decoder}

The structure of the three-stage serial-concatenated turbo encoder employed at the SNs and RNs is shown in Fig. 2, where 


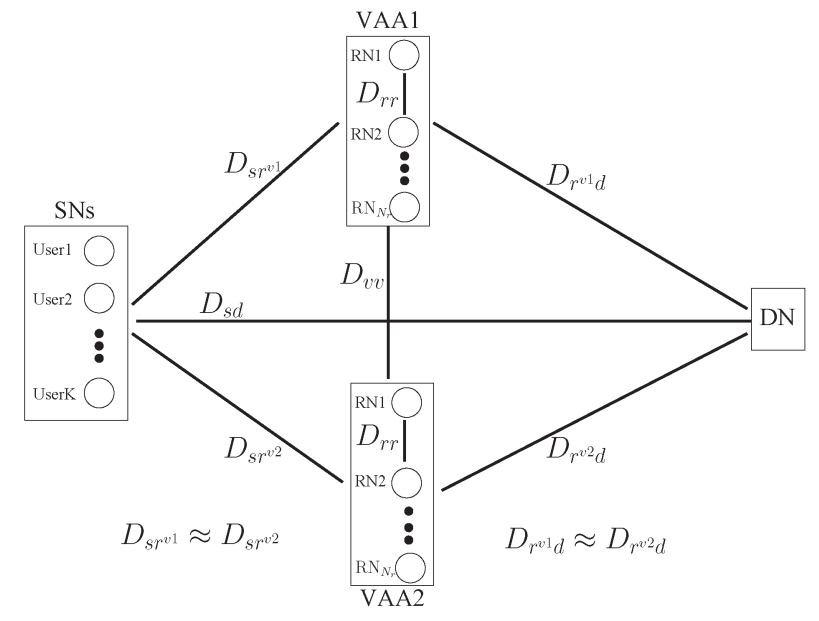

Fig. 3. Topology of the proposed MUSRC system. The system is symmetric in the sense that the distance from the SNs to the VAA1 is approximately equal to the distance from the SNs to the VAA2, whereas the distance between the VAA1 and the DN is approximately equal to the distance between the VAA2 and the DN.

the two-stage inner encoder is formed by a differential modulator (DM) combined with a URC encoder. The explicit benefit of incorporating a low-complexity memory-1 URC is that it has an infinite impulse response (IIR), which allows the system to spread the extrinsic information beneficially across the iterative decoder components without increasing its delay. As a result, a vanishingly low BER may be attained. Additionally, a half-rate RSC is employed as the outer encoder. This way, a three-stage RSC-URC-DM encoder is created.

The schematic of the corresponding three-stage turbo decoder adopted at the RNs and the DN is also portrayed in Fig. 2, which consists of a DPSK/DSTSK SISO-MSDSD detector, a URC decoder, and an RSC decoder. More explicitly, the composite inner decoder is formed by the combined SISO-MSDSD detector and the URC decoder, where the associated a priori information and extrinsic information are first interleaved and exchanged at $I_{\text {inner }}$ times. The outer decoder is constituted by the RSC decoder, where the information gleaned from the inner decoder is iteratively exchanged at $I_{\text {outer }}$ times. Due to the IIR of the URC decoder, the extrinsic information transfer (EXIT) curve is capable of reaching the $(1.0,1.0)$ point of perfect convergence to a vanishingly low BER in the EXIT charts (e.g., Figs. 6 and 11 in our simulation results), implying that no error floor occurs [35]. Therefore, again, the convergence performance of the system is improved by the URC decoder.

\section{Relay Architecture Overview}

We assume that the proposed MUSRC has a symmetric topology, as shown in Fig. 3. By exploiting the fact that the RNs in each VAA are geometrically close to each other and the distances between them are significantly smaller than the distances from the SNs to RNs and the RNs to DN, we can reasonably assume that the distances from the SNs to the RNs in a VAA are equal, and the distances spanning from the RNs in a VAA to the DN are also equal, which are denoted as $D_{s r}$ and $D_{r d}$, respectively. Then, the average path-loss reduction of the source-to-relay (SR) links and relay-to-destination (RD) links can be expressed as

$$
G_{s r}=\left(\frac{D_{s d}}{D_{s r}}\right)^{\alpha} \quad \text { and } \quad G_{r d}=\left(\frac{D_{s d}}{D_{r d}}\right)^{\alpha}
$$

respectively, where $D_{s d}$ is the distance between the SNs and $\mathrm{DN}$, which is normalized to unity. Additionally, the average path-loss reduction between the two VAAs is denoted as

$$
G_{v v}=\left(\frac{D_{s d}}{D_{v v}}\right)^{\alpha}
$$

where $D_{v v}$ is the distance between the two VAAs. Throughout this paper, the path-loss exponent is given by $\alpha=3$, which represents a typical urban area. Naturally, the given assumptions do not affect the general applicability of our analysis.

Since the direct signals transmitted from the SNs to the DN are attenuated by the pathloss, particularly in high-carrierfrequency mobile broadband systems, such as millimeter-wave communication and free-space optical mobile systems [36], [37], we only consider the SR and RD transmissions. For the SR transmission, we adopt DPSK signaling (e.g., DBPSK and DQPSK), while for the RD transmission, we adopt the $\operatorname{DSTSK}\left(N_{r}, N, T, Q, \mathcal{L}\right)$ system associated with $N_{r}=T$. Naturally, increasing $T$ improves the achievable BER performance at the cost of reducing the system throughput while imposing higher complexity in forming a VAA [34]. Having higher complexity is due to the fact that, since $N_{r}=T$, the designer has to choose more RNs, and the synchronization of the RNs also becomes more of a challenge. Therefore, we limit the number of the time slots to $T=2$ and mainly consider two configurations, namely $\operatorname{DSTSK}(2,1,2, Q, \mathcal{L})$ and $\operatorname{DSTSK}(2,2,2, Q, \mathcal{L})$, associated with $N=1$ and $N=2$ receive antennas at the DN, respectively.

Since no CSI is required by our MUSRC, no CSI is available for selecting RNs. Hence, the selection of RNs can only be based on their geometric location information [11]. With reference to Fig. 3, let us now discuss how to select the RNs and how to arrange the selected RNs into the two VAAs, based on the RNs' geometric location information. It is plausible that having a symmetric topology, as shown in Fig. 3, is reasonable, because the two VAAs may be identically treated. In this symmetric topology, we can denote the distance from the SNs to each of the two VAAs as $D_{s r}$ while denoting the distance between each of the two VAAs and the DN as $D_{r d}$. To benefit from collaborative relaying, we have

$$
D_{s r}<D_{s d} \quad \text { and } \quad D_{r d}<D_{s d} .
$$

The distance between any pair of RNs in a VAA, which is denoted as $D_{r r}$, satisfies

$$
D_{r r} \ll D_{s r} \quad \text { and } \quad D_{r r} \ll D_{r d} .
$$

Furthermore, we should have $D_{r r} \geq 10 \times \lambda$, where $\lambda$ is the carrier's wavelength, to ensure that we fully exploit the spatial diversity, but this condition is usually automatically met for the mobile relays. Regarding how to divide the selected RNs into two VAAs, we note that the distance between the two 
VAAs, which is denoted as $D_{v v}$, should be sufficiently high, so that the IVA is minimized. On the other hand, $D_{v v}$ should not be excessive so that the condition (1) is satisfied. Finally, the question of whether we should place a VAA closer to the SNs or closer to the DN arises. Since the RD links employ DSTSK, which has an inherent diversity capability and it is more powerful than the DPSK scheme employed by the SR links, therefore, we may appoint a VAA closer to the SNs, for the sake of achieving a balance of performance for the SR and RD links. This would also mitigate the error propagation inflicted by the DF scheme. More explicitly, we arrange for

$$
D_{s r}<D_{r d} .
$$

Let us now detail the operations involved in SNs, RNs, and DN.

\section{SNS}

During the broadcast interval, the information bit sequence $\mathbf{b}^{k}$ of the $k$ th user is encoded by the two-stage serialconcatenated channel encoder of Fig. 2, and the encoded bit sequence $\mathbf{u}^{k}$ is mapped to a PSK symbol sequence $\mathbf{x}^{k}=\left\{x_{n}^{k}\right\}$, which is then differentially encoded according to

$$
s_{n}^{k}= \begin{cases}1, & n=0 \\ x_{n}^{k} \cdot s_{n-1}^{k}, & n>0 .\end{cases}
$$

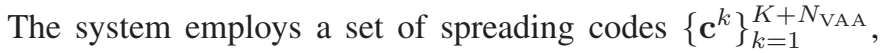
each having a spreading factor of $L_{s}$, where $N_{\mathrm{VAA}}=2$ is the number of VAAs. The first $K$ spreading codes are used for supporting the $K$ users, and the spreading operation at the $k$ th $\mathrm{SN}$ is expressed as

$$
\mathbf{e}_{n}^{k}=\frac{1}{\sqrt{L_{s}}} s_{n}^{k} \mathbf{c}^{k}, \quad 1 \leq k \leq K .
$$

\section{E. RNS}

We assume that the CDMA up-link is synchronized, so that all the signals arrive at a $\mathrm{RN}$ with the same delay; hence, the near-zero cross-correlation property of the DS-CDMA system may be exploited for reducing the multiuser interference (MUI). Additionally, the Rayleigh fading channel is considered to be quasi-static, yielding a constant fading envelope and phase within an $L_{s}$-chip transmission period. Let the two VAAs be denoted by $V_{1}$ and $V_{2}$, respectively. In the sequel, a columnvector-based notation will be adopted.

1) Decoding: The signal received at the $m$ th $\mathrm{RN}$ of the VAA $V_{1}$ may be expressed as

$$
\begin{aligned}
\mathbf{y}_{n V_{1}}^{m}= & \sqrt{G_{s r}} \sum_{k=1}^{K} \mathbf{e}_{n}^{k} h_{n V_{1}}^{k m} \\
& +\sqrt{G_{v v}}\left\{\text { first }-L_{s} \text {-elements }\left(\mathbf{A}_{V_{2}} \mathbf{H}_{V_{2}}^{m}\right)\right\}+\overline{\mathbf{v}}_{n V_{1}}^{m}
\end{aligned}
$$

where $\mathbf{y}_{n V_{1}}^{m} \in \mathbb{C}^{L_{s} \times 1}, h_{n V_{1}}^{k m} \in \mathbb{C}$ is the $n$th Rayleigh distributed channel coefficient between the $k$ th SN and the $m$ th RN of the VAA $V_{1}$, and $\mathbf{A}_{V_{2}} \in \mathbb{C}^{T L_{s} \times N_{r}}$ is the received CDMA-spread DSTSK signal block from the VAA $V_{2} . \mathbf{H}_{V_{2}}^{m} \in \mathbb{C}^{N_{r} \times 1}$ is the equivalent channel matrix between the VAA $V_{2}$ and the $m$ th RN of the VAA $V_{1}$, whereas $\overline{\mathbf{v}}_{n V_{1}}^{m} \in \mathbb{C}^{L_{s} \times 1}$ is the equivalent additive white Gaussian noise (AWGN) vector. Despreading $\mathbf{y}_{n V_{1}}^{m}$ for the $k$ th user yields

$$
\begin{aligned}
r_{n V_{1}}^{k m} & =\frac{\sqrt{G_{s r}}}{\sqrt{L_{s}}}\left(\mathbf{c}^{k}\right)^{T} \mathbf{y}_{n V_{1}}^{m} \\
& =\frac{\sqrt{G_{s r}}}{\sqrt{L_{s}}}\left(\mathbf{c}^{k}\right)^{T} \mathbf{e}_{n}^{k} h_{n V_{1}}^{k m}+\eta_{n V_{1}}^{k m}+\bar{v}_{n V_{1}}^{k m} \\
& =\sqrt{G_{s r}} s_{n}^{k} h_{n V_{1}}^{k m}+\eta_{n V_{1}}^{k m}+\bar{v}_{n V_{1}}^{k m}
\end{aligned}
$$

where $\eta_{n V_{1}}^{k m}$ denotes the MUI and IVI terms, which may be substantially reduced as a benefit of the near-orthogonality of the spreading code family employed [30], and $\bar{v}_{n V_{1}}^{k m}$ is the noise term having a zero mean and a variance of $N_{0} / 2$ per dimension. When considering a detection window of $N_{w}$ received samples, (7) may be expressed as

$$
\mathbf{r}=\sqrt{G_{s r}} \operatorname{diag}\{\mathbf{s}\} \mathbf{h}+\mathbf{v}=\sqrt{G_{s r}} \mathbf{S h}+\mathbf{v}
$$

where $\mathbf{r}=\left[r_{\left(n-N_{w}+1\right) V_{1}}^{k m} r_{\left(n-N_{w}+2\right) V_{1}}^{k m} \cdots r_{n V_{1}}^{k m}\right]^{T}$ includes the $N_{w}$ consecutive signals received within the detection window of $N_{w}$ samples, $\mathbf{s}=\left[s_{n-N_{w}+1}^{k} s_{n-N_{w}+2}^{k} \cdots s_{n}^{k}\right]^{T}$ is the corresponding differentially encoded symbol vector, $\mathbf{S}=\operatorname{diag}\{\mathbf{s}\}$, $\mathbf{h} \in \mathbb{C}^{N_{w} \times 1}$ is the Rayleigh fading channel vector, and $\mathbf{v} \in$ $\mathbb{C}^{N_{w} \times 1}$ the AWGN vector. Note that $\mathbf{S}$ is a unitary matrix, and any small residual MUI and IVI term in (7) is assumed to be included in $\mathbf{v}$.

The SISO-MSDSD is based on the Log-MAP algorithm [35], where the multiple-symbol decisions depend on the evaluation of the conditional probability density function (pdf) $p(\mathbf{r} \mid \mathbf{s})$, which satisfies the following relation [29]:

$$
-\ln (p(\mathbf{r} \mid \mathbf{s})) \propto \mathbf{r}^{H} \mathbf{R}_{r r}^{-1} \mathbf{r} .
$$

If we define the channel's correlation matrix as $\mathbf{R}_{h h}=$ $\varepsilon\left\{\mathbf{h} \mathbf{h}^{H}\right\}$, the noise correlation matrix as $\mathbf{R}_{v v}=\varepsilon\left\{\mathbf{v} \mathbf{v}^{H}\right\}$ and the combined correlation matrix $\mathbf{C}=\mathbf{R}_{h h}+\left(1 / G_{s r}\right) \mathbf{R}_{v v}$, then the power normalized conditional correlation matrix $\mathbf{R}_{r r}$ of the received signal vector $\mathbf{r}$ in (9) may be expressed as

$$
\mathbf{R}_{r r}=\frac{1}{G_{s r}} \varepsilon\left\{\mathbf{r r}^{H}\right\}=\mathbf{S R}_{h h} \mathbf{S}^{H}+\frac{1}{G_{s r}} \mathbf{R}_{v v}=\mathbf{S C S}^{H} .
$$

Upon applying the Cholesky factorization, we have $\mathbf{C}^{-1}=$ $\mathbf{L} \mathbf{L}^{H}$, where $\mathbf{L}$ is a lower triangular matrix. By defining the upper triangular matrix of

$$
\mathbf{U}=\left(\frac{1}{\sqrt{G_{s r}}} \mathbf{L}^{H} \operatorname{diag}\{\mathbf{r}\}\right)^{*}
$$

we arrive at

$$
-\ln (p(\mathbf{r} \mid \mathbf{s})) \propto\|\mathbf{U} \mathbf{s}\|^{2} .
$$

Note that a common phase shift of all the elements in the differential symbol vector $\mathbf{s}$ has no effect on the ML metric of 
(12). If we define the accumulated differential symbol as [38]

$$
\begin{aligned}
a_{i} & = \begin{cases}\prod_{j=i}^{N_{w}-1}\left(x_{j}\right)^{*}, & 1 \leq i \leq N_{w}-1 \\
1, & i=N_{w}\end{cases} \\
& = \begin{cases}x_{i}^{*} a_{i+1}, & 1 \leq i \leq N_{w}-1 \\
1, & i=N_{w} .\end{cases}
\end{aligned}
$$

the following relationship may be obtained:

$$
-\ln (p(\mathbf{r} \mid \mathbf{s})) \propto\|\mathbf{U} \mathbf{a}\|^{2}
$$

where $\mathbf{a}=\left[a_{1} a_{2} \cdots a_{N_{w}}\right]^{T}$ is the accumulated differential symbol vector. Note that the user index ${ }^{k}$ has been omitted from $a_{i}$ and $x_{j}$ for notational simplicity. Sphere decoding (SD) examines the set of candidates $\left\{x_{j}\right\}_{j=1}^{N_{w}-1}$ that lie within the decoding sphere radius $R$, i.e., within

$$
\|\mathbf{U} \mathbf{a}\|^{2} \leq R^{2} .
$$

The SISO-MSDSD accepts the a priori soft information from the URC decoder of Fig. 2 and produces the corresponding soft outputs. Assuming that the symbols $\left\{x_{j}\right\}_{j=1}^{N_{w}-1}$ are independent, their a priori information may be expressed as

$$
\ln (\operatorname{Pr}\{\mathbf{x}\})=\sum_{j=1}^{N_{w}-1} \ln \left(\operatorname{Pr}\left\{x_{j}\right\}\right)
$$

and we may further manipulate (15) to yield

$$
\begin{aligned}
\|\mathbf{U} \mathbf{a}\|^{2} & -\ln (\operatorname{Pr}\{\mathbf{x}\}) \\
& =\sum_{j=1}^{N_{w}-1}\left(\left|\sum_{l=j}^{N_{w}-1} u_{j l} a_{l}\right|^{2}-\ln \left(\operatorname{Pr}\left\{x_{j}\right\}\right)\right) \leq R^{2} .
\end{aligned}
$$

The partial-Euclidean-distance (PED) contribution of each bit of the DSTSK symbol may then be defined as

$$
d_{i}^{2}=\sum_{j=i}^{N_{w}-1}\left(\left|\sum_{l=j}^{N_{w}-1} u_{j l} a_{l}\right|^{2}-\ln \left(\operatorname{Pr}\left\{x_{j}\right\}\right)\right)=d_{i+1}^{2}+\Delta_{i}^{2}
$$

where the PED increment may be expressed as

$$
\Delta_{i}^{2}=\left|u_{i i} a_{i+1} x_{i}^{*}+\sum_{l=i+1}^{N_{w}-1} u_{i l} a_{l}\right|^{2}-\ln \left(\operatorname{Pr}\left\{x_{i}\right\}\right) .
$$

Assuming that the symbol $x_{n}$ is detected, the a posteriori probability for the SISO-MSDSD may be calculated by the Max-Log-MAP algorithm [35], which is expressed as

$$
\begin{aligned}
L_{p}\left(b_{n} \mid \mathbf{r}\right)= & \ln \left(\frac{\operatorname{Pr}\left\{b_{n}=1 \mid \mathbf{r}\right\}}{\operatorname{Pr}\left\{b_{n}=0 \mid \mathbf{r}\right\}}\right) \approx-\left\|\mathbf{U} \mathbf{a}_{\mathrm{MAP}}^{b_{n}=1}\right\|^{2} \\
& +\ln \left(\operatorname{Pr}\left\{x_{\mathrm{MAP}}^{b_{n}=1}\right\}\right)+\left\|\mathbf{U} \mathbf{a}_{\mathrm{MAP}}^{b_{n}=0}\right\|^{2} \\
& -\ln \left(\operatorname{Pr}\left\{x_{\mathrm{MAP}}^{b_{n}=0}\right\}\right) \\
= & d_{\mathrm{MAP}}^{b_{n}=1}-d_{\mathrm{MAP}}^{b_{n}=0}
\end{aligned}
$$

TABLE I

COMBINATIONS OF $Q$ AND $\mathcal{L}$ FOR SUPPORTING DiFFERENT NuMBERS OF USERS $K$ AND THE CORRESPONDING LOWER BOUND COMPLEXITY $N_{c}$ [28] Associated With $N_{w}=4$, DBPSK at SNs, AND $N=1$ RECEIVE ANTENNA AT THE DN

\begin{tabular}{|c|c|c|c|}
\hline$K=2$ & $K=3$ & $K=4$ & $K=5$ \\
\hline $\begin{array}{c}Q=2, \mathcal{L}=2 \\
\left(N_{c}=180\right)\end{array}$ & $\begin{array}{c}Q=2, \mathcal{L}=4 \\
\left(N_{c}=200\right)\end{array}$ & $\begin{array}{c}Q=2, \mathcal{L}=8 \\
\left(N_{c}=250\right)\end{array}$ & $\begin{array}{c}Q=2, \mathcal{L}=16 \\
\left(N_{c}=360\right)\end{array}$ \\
\hline N/A & $\begin{array}{c}Q=4, \mathcal{L}=2 \\
\left(N_{c}=200\right)\end{array}$ & $\begin{array}{c}Q=4, \mathcal{L}=4 \\
\left(N_{c}=250\right)\end{array}$ & $\begin{array}{c}Q=4, \mathcal{L}=8 \\
\left(N_{c}=360\right)\end{array}$ \\
\hline N/A & N/A & $\begin{array}{c}Q=8, \mathcal{L}=2 \\
\left(N_{c}=250\right)\end{array}$ & $\begin{array}{c}Q=8, \mathcal{L}=4 \\
\left(N_{c}=360\right)\end{array}$ \\
\hline N/A & N/A & N/A & $\begin{array}{c}Q=16, \mathcal{L}=2 \\
\left(N_{c}=360\right)\end{array}$ \\
\hline
\end{tabular}

where $d_{\mathrm{MAP}}^{b_{n}=1}$ and $d_{\mathrm{MAP}}^{b_{n}=0}$ indicate the minimum PEDs estimated by the SD when the bit of interest $b_{n}$ is set to 1 and 0 , respectively. Again, the user index ${ }^{k}$ has been omitted from $b_{n}$.

The extrinsic information of $b_{n}$ may be calculated by subtracting the a priori information of (16) from the a posteriori probability of (20), which can then be fed to the URC decoder to form an decoding inner loop, as portrayed in Fig. 2. Finally, after the termination of the three-stage MSDSD-URC-RSC turbo decoding process depicted in Fig. 2, the hard-decision outputs of the three-stage turbo decoder recover the bit sequences of the $K$ users at the $m$ th $\mathrm{RN}$, each having the length $L_{f}$, to form the decision matrix $\widehat{\mathbf{B}}^{m}=\left[\widehat{\mathbf{b}}^{1 m} \widehat{\mathbf{b}}^{2 m} \cdots \widehat{\mathbf{b}}^{K m}\right]$, where we have $m \in\left\{1, \ldots, N_{r}\right\}$ in our system. The details of the three-stage turbo decoding process can be found in [35].

2) Forwarding: To perform the three-stage serialconcatenated RSC-URC-DSTSK encoding, as shown in Fig. 2, the decision matrix $\widehat{\mathbf{B}}^{m} \in \mathbb{C}^{L_{f} \times K}$ is first parallel-to-serial converted to form the decision vector $\widehat{\mathbf{b}}_{\text {vec }}^{m} \in \mathbb{C}^{K L_{f} \times 1}$. Then, this decision vector is encoded by the half-rate RSC-URC encoder to generate a coded sequence $\widehat{\mathbf{u}}^{m} \in \mathbb{C}^{2 K L_{f} \times 1}$, which is then serial-to-parallel converted to the coded matrix $\widehat{\mathbf{U}}^{m} \in$ $\mathbb{C}^{2 L_{f} \times K}$ and then transmitted by the DSTSK modulator [39].

To support $K$ users, the number of bits conveyed by each DSTSK signal block and the number of users has to meet the following condition:

$$
K=\log _{2}(Q)+\log _{2}(\mathcal{L})
$$

where $\log _{2}(Q)$ bits are used for choosing a specific dispersion matrix $\mathbf{D}_{q} \in \mathbb{C}^{T \times N_{r}}$ from the set of $Q$ dispersion matrices, and $\log _{2}(\mathcal{L})$ bits are adopted to unambiguously represent a specific constellation point $s_{l}(n)$ of the conventional $\mathcal{L}$-PSK modulation scheme. To elaborate a little further, supporting different numbers of users can be realized by appropriately modifying the values of $Q$ and $\mathcal{L}$, without changing the system configuration. For example, we may have $Q=2$ and $\mathcal{L}=2$ for a system supporting $K=2$ users, whereas we may opt for the values of $Q=4$ and $\mathcal{L}=4$ for supporting $K=4$ users. Additionally, given a specific number of users, various combinations of $Q$ and $\mathcal{L}$ may be used, provided that the relation (21) is satisfied, which are summarized in Table I for different values of $K$. The most beneficial DSTSK combination selection is detailed in Section III.

After obtaining the dispersion matrix $\mathbf{D}_{q}$ and the corresponding conventional $\mathcal{L}$-PSK symbol $s_{l}(n)$, the STSK signal block 
is generated as

$$
\mathbf{X}_{n}^{m}=s_{l}(n) \mathbf{D}_{q}
$$

The number of transmit antennas and the number of time slots should be identical for the DSTSK scheme [34], i.e., $N_{r}=T$. With this constraint, the DSTSK symbol block is given by

$$
\mathbf{S}_{n}^{m}= \begin{cases}\mathbf{I}_{T}, & n=0 \\ \mathbf{X}_{n}^{m} \mathbf{S}_{n-1}^{m}, & n>0\end{cases}
$$

which is spread by the spreading code $\mathbf{c}_{V_{1}}$ of the VAA $V_{1}$ to yield

$$
\mathbf{A}_{V_{1}}^{m}=\frac{1}{\sqrt{L_{s}}} \mathbf{S}_{n}^{m} \otimes \mathbf{c}_{V_{1}}
$$

where the spread DSTSK signal block obeys $\mathbf{A}_{V_{1}}^{m} \in \mathbb{C}^{T L_{s} \times N_{r}}$. Since, in our proposed MUSRC system, $N_{r}$ RNs form a VAA, if we denote the equivalent signal block transmitted by the VAA $V_{1}$ to the $\mathrm{DN}$ as $\mathbf{A}_{V_{1}} \in \mathbb{C}^{T L_{s} \times N_{r}}$, then the $m$ th column of $\mathbf{A}_{V_{1}}$ is contributed by the $m$ th column of $\mathbf{A}_{V_{1}}^{m}$. To be more specific, the $m$ th column of $\mathbf{A}_{V_{1}}^{m}$ is transmitted by the corresponding $m$ th $\mathrm{RN}$ to the DN. This corresponds to the system block diagram shown in Fig. 1, where for example, the first RN in the VAA1 extracts and transmits the first column of the DSTSKmodulated and spread signal block matrix formulated in (24). Note that the allocation of the total transmission power to the RNs in a VAA is automatically performed. This is because every dispersion matrix $\mathbf{D}_{q}$ is designed to have the same constant power [34]; therefore, every equivalent signal matrix $\mathbf{A}_{V_{1}}$ has the same constant power. Since the $m$ th RN transmits the $m$ th column of $\mathbf{A}_{V_{1}}$, where $1 \leq m \leq N_{r}$, the transmit power of all the $N_{r}$ RNs adds up to the total transmit power. Interestingly, the DSTSK scheme of (23) may be viewed as the generalization of the DPSK arrangement of (4) by replacing the scalars in (4) with matrices.

\section{F. DN}

All the RNs are assumed to be perfectly synchronized, implying that during the $T$ time slots, the $N_{r}$ RNs in a specific VAA group transmit their signal blocks simultaneously. Furthermore, the Rayleigh fading channel is assumed to be quasi-static so that the fading envelope and phase remain constant within the time duration of $L_{s} T$. The despread signal block received at the DN with the aid of $N$ receive antennas over $T$ time slots may be expressed as [34]

$$
\mathbf{R}_{n}=\sqrt{G_{r d}} \mathbf{S}_{n} \mathbf{H}_{n}+\mathbf{V}_{n}
$$

where we have $\mathbf{R}_{n} \in \mathbb{C}^{T \times N} ; \mathbf{S}_{n} \in \mathbb{C}^{T \times N_{r}}$ is the equivalent differential signal block received from the transmitting VAA, whose $m$ th column is identical to the $m$ th column of $\mathbf{S}_{n}^{m}$; $\mathbf{H}_{n} \in \mathbb{C}^{N_{r} \times N}$ denotes the fading channel matrix between the transmitting VAA and the DN; and $\mathbf{V}_{n} \in \mathbb{C}^{T \times N}$ is the corresponding AWGN matrix. Note the "similarity" between (25) and (7), where the scalar elements in (7) are replaced by matrices in (25). Unlike (7), however, there exists no MUI or IVI in (25).

Similar to the RNs, the three-stage MSDSD-URC-RSC turbo decoder detects the signal blocks received at the DN. More explicitly, the URC and RSC decoders of Fig. 2 used at the DN are exactly identical to those employed in the RNs, whereas the SISO-MSDSD of the RNs is extended for detecting the DSTSK signal blocks. In particular, over the $N_{w}$ DSTSK signal blocks, (25) can be expressed as

$$
\overline{\mathbf{R}}=\sqrt{G_{r d}} \overline{\mathbf{S H}}+\overline{\mathbf{V}}
$$

where we have $\overline{\mathbf{R}}=\left[\mathbf{R}_{n-N_{w}+1}^{T} \mathbf{R}_{n-N_{w}+2}^{T} \cdots \mathbf{R}_{n}^{T}\right]^{T}$

$$
\overline{\mathbf{S}}=\left[\begin{array}{cccc}
\mathbf{S}_{n-N_{w}+1} & \mathbf{0} & \cdots & \mathbf{0} \\
\mathbf{0} & \mathbf{S}_{n-N_{w}+2} & \ddots & \vdots \\
\vdots & \ddots & \ddots & \mathbf{0} \\
\mathbf{0} & \cdots & \mathbf{0} & \mathbf{S}_{n}
\end{array}\right]
$$

$\overline{\mathbf{H}}=\left[\mathbf{H}_{n-N_{w}+1}^{T} \mathbf{H}_{n-N_{w}+2}^{T} \cdots \mathbf{H}_{n}^{T}\right]^{T} \quad$ and $\quad \overline{\mathbf{V}}=\left[\mathbf{V}_{n-N_{w}+1}^{T}\right.$ $\left.\mathbf{V}_{n-N_{w}+2}^{T} \cdots \mathbf{V}_{n}^{T}\right]^{T}$. We emphasize again the "similarity" between (26) and (8), where the essential operational difference of the SISO-MSDSD invoked for DPSK and for DSTSK is in the mapping of bits to DPSK symbols $s_{n}$ and the mapping of bits to the DSTSK signal blocks $\mathbf{S}_{n}$. In other words, apart from this difference, the operation of the threestage MSDSD-URC-RSC turbo decoding process invoked for the DSTSK scheme may be considered to be identical to that of DPSK, as detailed in Section II-B. We point out furthermore that the three-stage MSDSD-URC-RSC turbo decoding algorithm of DSTSK employed by the DN has the same form as that of the three-stage MSDSD-URC-RSC turbo decoder conceived for the single-user differential-space-timeblock-code-based relaying system [38]. The DN applies this three-stage MSDSD-URC-RSC turbo decoding algorithm for generating the bit decisions for the $K$ users.

\section{Differential Space-Time Shift Keying Configuration SElEction}

In DSTSK schemes, the choice of the dispersion matrix set $\mathbf{D}_{q}, 1 \leq q \leq Q$ has significant effects on the system's achievable performance [34].

\section{A. Dispersion Matrix Generation}

The design or generation of a near-optimum DSTSK dispersion matrix set was proposed in [39], which is based on a carefully conducted random search. To elaborate a little further, the dispersion matrices $\mathbf{D}_{q}, 1 \leq q \leq Q$ are first randomly generated as unitary matrices, which obey the power constraint of

$$
\operatorname{tr}\left[\mathbf{D}_{q}^{H} \mathbf{D}_{q}\right]=T, \quad 1 \leq q \leq Q
$$

hence leading to a unity average transmission power for each DSTSK symbol duration. Then, these initial DSTSK symbol matrices are optimized using a random search algorithm to 
TABLE II

MAXimum-Minimum-DeterminAnt $d_{\max }-\min (Q, \mathcal{L})$ FOR DifFEREnt Combinations of $(Q, \mathcal{L})$

\begin{tabular}{|c|c|c|c|c|}
\hline \multicolumn{5}{|c|}{ DBPSK at SNs and $N=1$ receive antenna at DN } \\
\hline & $Q=2$ & $Q=4$ & $Q=8$ & $Q=16$ \\
\hline $\mathcal{L}=2$ & $d_{\max -\min }(2,2)=1.99846$ & $d_{\max -\min }(4,2)=1.41065$ & $d_{\max -\min }(8,2)=0.47171$ & $d_{\max -\min }(16,2)=0.092138$ \\
\hline $\mathcal{L}=4$ & $d_{\max -\min }(2,4)=1.21914$ & $d_{\max -\min }(4,4)=0.75919$ & $d_{\max -\min }(8,4)=0.21126$ & N/A \\
\hline $\mathcal{L}=8$ & $d_{\max -\min }(2,8)=0.58578$ & $d_{\max -\min }(4,8)=0.40317$ & N/A & N/A \\
\hline $\mathcal{L}=16$ & $d_{\max -\min }(2,16)=0.15224$ & N/A & N/A & N/A \\
\hline \multicolumn{5}{|c|}{ DQPSK at SNs and $N=2$ receive antennas at DN } \\
\hline & $Q=2$ & $Q=4$ & $Q=8$ & $Q=16$ \\
\hline $\mathcal{L}=2$ & $d_{\max -\min }(2,2)=1.99822$ & $d_{\max -\min }(4,2)=1.41172$ & $d_{\max -\min }(8,2)=0.42625$ & $d_{\max -\min }(16,2)=0.10477$ \\
\hline $\mathcal{L}=4$ & $d_{\max -\min }(2,4)=1.36047$ & $d_{\max -\min }(4,4)=0.80871$ & $d_{\max -\min }(8,4)=0.20731$ & N/A \\
\hline $\mathcal{L}=8$ & $d_{\max -\min }(2,8)=0.58579$ & $d_{\max -\min }(4,8)=0.42085$ & N/A & N/A \\
\hline $\mathcal{L}=16$ & $d_{\max -\min }(2,16)=0.15224$ & N/A & N/A & N/A \\
\hline
\end{tabular}

minimize the pairwise symbol error probability (PSEP) expressed as [39]

$$
p(\mathbf{X} \rightarrow \hat{X}) \leq \frac{1}{\operatorname{det}\left(\mathbf{I}_{T N}+\frac{E_{s}}{4 N_{0}} \mathbf{R}_{\mathbf{X}} \otimes \mathbf{I}_{N}\right)}
$$

where $T$ and $N$ are the numbers of time slots and receive antennas, respectively; $E_{s}$ denotes the symbol energy; and $N_{0}$ the AWGN power. Additionally, $\mathbf{R}_{\mathbf{X}}$ in (28) is the codeword difference matrix, which may be expressed as $\mathbf{R}_{\mathbf{X}}=(\mathbf{X}-$ $\hat{\mathbf{X}})^{H}(\mathbf{X}-\hat{X})$, where $\mathbf{X}$ and $\hat{\mathbf{X}}$ are an arbitrary pair of the legitimate codewords selected from the DSTSK symbol set having a size of $Q \cdot \mathcal{L}$.

In the PSEP expression, if $\mathbf{R}_{\mathbf{X}}$ has full rank, the error probability will be determined by the minimum value of the determinant of $\mathbf{R}_{\mathbf{X}}$ [40]. As a result, by ensuring that $\mathbf{R}_{\mathbf{X}}$ has full rank, the DSTSK dispersion matrix set may be optimized by maximizing the minimum determinant $d_{\min }$ of $\mathbf{R}_{\mathbf{X}}$ for any pair of legitimate codewords, where $d_{\min }$ is the function of $\mathbf{D}_{q}$, $1 \leq q \leq Q$, and $\mathcal{L}$, which can be expressed as

$$
d_{\min }\left(\mathbf{D}_{q}, 1 \leq q \leq Q ; \mathcal{L}\right)=\min \left\{\operatorname{det}\left[\mathbf{R}_{\mathbf{X}}\right], \quad \forall \mathbf{R}_{\mathbf{X}}\right\}
$$

In other words, given a legitimate configuration $Q$ and $\mathcal{L}$, the set of near-optimum DSTSK dispersion matrices can be determined by solving the optimization

$$
d_{\max -\min }(Q, \mathcal{L})=\max _{\substack{\mathbf{D}_{q}, 1 \leq q \leq Q \\ \log _{2}(Q \cdot \mathcal{L})=K}} d_{\min }\left(\mathbf{D}_{q}, 1 \leq q \leq Q ; \mathcal{L}\right)
$$

\section{B. $M M B C S$}

It has been mentioned in Section II-E2 that the proposed MUSRC system is capable of supporting the same number of users by different DSTSK configurations. To be more explicit, according to (21), for a given number of users $K$, we may opt for different combinations of $Q$ and $\mathcal{L}$. For example, if we have $K=4$ users, the possible combinations of $Q$ and $\mathcal{L}$ are $(Q, \mathcal{L})=(2,8),(Q, \mathcal{L})=(4,4)$, and $(Q, \mathcal{L})=(8,2)$. A detailed DSTSK configuration list is shown in Table I. Since different configurations $(Q, \mathcal{L})$ for the same number of users $K$ may have different $d_{\max -\min }(Q, \mathcal{L})$, the resultant BERs may also be different. The most appropriate DSTSK configuration
TABLE III

Optimal Combinations $\left(Q_{\text {opt }}, \mathcal{L}_{\text {opt }}\right)$ FOR SUPPORTING DifFERENT NUMBERS OF USERS $K$, ASSOCIATED WITH DBPSK OR DQPSK AT SNS AND $N=1$ OR Two RECEIVE ANTENNAS AT THE DN

\begin{tabular}{|c|c|c|c|}
\hline$K=2$ & $K=3$ & $K=4$ & $K=5$ \\
\hline $\begin{array}{c}\left(Q_{\mathrm{opt}}, \mathcal{L}_{\mathrm{opt}}\right) \\
=(2,2)\end{array}$ & $\begin{array}{c}\left(Q_{\mathrm{opt}}, \mathcal{L}_{\mathrm{opt}}\right) \\
=(4,2)\end{array}$ & $\begin{array}{c}\left(Q_{\mathrm{opt}}, \mathcal{L}_{\mathrm{opt}}\right) \\
=(4,4)\end{array}$ & $\begin{array}{c}\left(Q_{\mathrm{opt}}, \mathcal{L}_{\mathrm{opt}}\right) \\
=(4,8)\end{array}$ \\
\hline
\end{tabular}

is obviously the one that yields the lowest BER. One way to choose the best configuration is to perform the Monte Carlosimulation-based BER calculations for all the legitimate configurations of the DSTSK system for a fixed number of users $K$ (i.e., a fixed throughput). However, the associated Monte Carlo simulations are very time-consuming. Therefore, we avoid such a Monte-Carlo-simulation-based approach and select the best DSTSK configuration based on the maximum-minimum determinant $d_{\text {min }}$ detailed in Section III-A, which leads to our proposed MMBCS algorithm.

More explicitly, given a fixed throughput, for each of the legitimate DSTSK configuration $(Q, \mathcal{L})$, the near-optimum set of dispersion matrices is generated by solving the optimization (30), which also records the corresponding maximumminimum-determinant value $d_{\max -\min }(Q, \mathcal{L})$. The optimal DSTSK configuration $\left(Q_{\text {opt }}, \mathcal{L}_{\text {opt }}\right)$ is then simply chosen as

$$
\left(Q_{\text {opt }}, \mathcal{L}_{\text {opt }}\right)=\arg \max _{Q, \mathcal{L}: \log _{2}(Q \cdot \mathcal{L})=K} d_{\max -\min }(Q, \mathcal{L}) .
$$

The maximum-minimum-determinant values of $d_{\max -\min }(Q, \mathcal{L})$ for various DSTSK configurations $(Q, \mathcal{L})$ are listed in Table II for the DBPSK system at the SNs with an $N=1$ receive antenna at the DN and the DQPSK system at the SNs with $N=2$ receive antennas at the DN. Further taking into account the results of Table I, we arrive at the optimal DSTSK configurations $\left(Q_{\text {opt }}, \mathcal{L}_{\text {opt }}\right)$ to support the various numbers of users $K$, which are summarized in Table III. In the following simulation study, we will demonstrate that the most appropriate DSTSK configuration selected by our proposed MMBCS algorithm is capable of outperforming other DSTSK configurations, in terms of their maximum achievable rate.

\section{Simulation Results}

A quasi-static Rayleigh fading environment having a normalized Doppler frequency $f_{d}=0.01$ was considered, and an interleaver length of 10000 bits was used by the three-stage 
serial-concatenated turbo encoder/decoder. The generator polynomials of the RSC encoder were $G_{\mathrm{RSC}}=[1,0,1]_{2}$ and $G_{\mathrm{RSC}}^{r}=[1,1,1]_{2}$, whereas the generator polynomials of the URC encoder were $G_{\mathrm{URC}}=[1,0]_{2}$ and $G_{\mathrm{URC}}^{r}=[1,1]_{2}$, where $G_{\mathrm{RSC}}^{r}$ and $G_{\mathrm{URC}}^{r}$ are the feedback polynomials of the RSC and URC encoders, respectively. The numbers of inner iterations and outer iterations were $I_{\text {inner }}=2$ and $I_{\text {outer }}=4$. Unless otherwise stated, ${ }^{1}$ the distance between the two VAAs was $D_{v v}=(1 / 4) \cdot N_{r}=2 \mathrm{RNs}$ were employed in each of the two VAA groups, leading to a total of $2 N_{r}=4$ relays. Since the transmit signal power of all the simulated systems (SNs to RNs and RNs to DN) was normalized to unity, the equivalent SNR of the overall system was defined as $1 / N_{0}$, with $N_{0}$ being the equivalent system's AWGN power. Taking into account the path losses in (7) and (25), the noise power at an RN was set to $N_{0} / G_{s r}$, whereas the noise power at the DN was given by $N_{0} / G_{r d}$. The set of $K+2$ nonorthogonal random codes with the spreading factor $L_{s}=256$ was used to support multiple $K$ users and the two VAAs.

\section{A. DBPSK for SR Transmission and DSTSK $(2,1,2, Q, \mathcal{L})$ for RD Transmission}

We first consider the proposed DSTSK-aided MUSRC system employing DBPSK for the SR transmission and a DSTSK scheme of $\operatorname{DSTSK}(2,1,2, Q, \mathcal{L})$ for the $\mathrm{RD}$ transmission, associated with $N_{r}=2$ relays in each of the two VAA groups and a DN equipped with $N=1$ receive antenna. Various number of $Q$ dispersion matrices and $\mathcal{L}$-PSK constellations may be adopted by the DSTSK scheme at the RNs, depending on the number of users $K$, as listed in Table I. The most appropriate or optimal combination $\left(Q_{\text {opt }}, \mathcal{L}_{\text {opt }}\right)$ can be determined using the proposed MMBCS algorithm, as presented in Section III-B. The optimal combinations $\left(Q_{\text {opt }}, \mathcal{L}_{\text {opt }}\right)$ corresponding to various $K$ can be found in Table III. Therefore, we commenced our investigations with the aid of the maximum achievable rate to verify the facts that the different combinations of $(Q, \mathcal{L})$ lead to different system performances, and the optimal combination $\left(Q_{\text {opt }}, \mathcal{L}_{\text {opt }}\right)$ found by the MMBCS algorithm indeed offers the optimal performance. The simulation results obtained are shown in Fig. 4, where the DSTSK-aided MUSRC systems supporting $K \in\{3,4\}$ users were considered, in conjunction with an MSDSD window size of $N_{w}=4$. The SR distance was chosen as $D_{s r}=0.51$, which will be shown to be the optimal distance for the system configurations used here.

It is shown in Table I that, for $K=3$ users, there are two legitimate combinations of $(Q, \mathcal{L})=(4,2)$ and $(Q, \mathcal{L})=$ $(2,4)$. The corresponding maximum achievable rates of these two configurations are shown in Fig. 4 as two dashed curves, where it is seen that both these two DSTSK combinations are capable of achieving the system throughput of 3 bits/symbol at approximately SNR $=7 \mathrm{~dB}$. However, based on the fact that for near-capacity systems, a vanishingly low BER may occur

\footnotetext{
${ }^{1}$ Normally, the closer the two VAAs, the stronger IVI, and the poorer the achievable system performance.
}

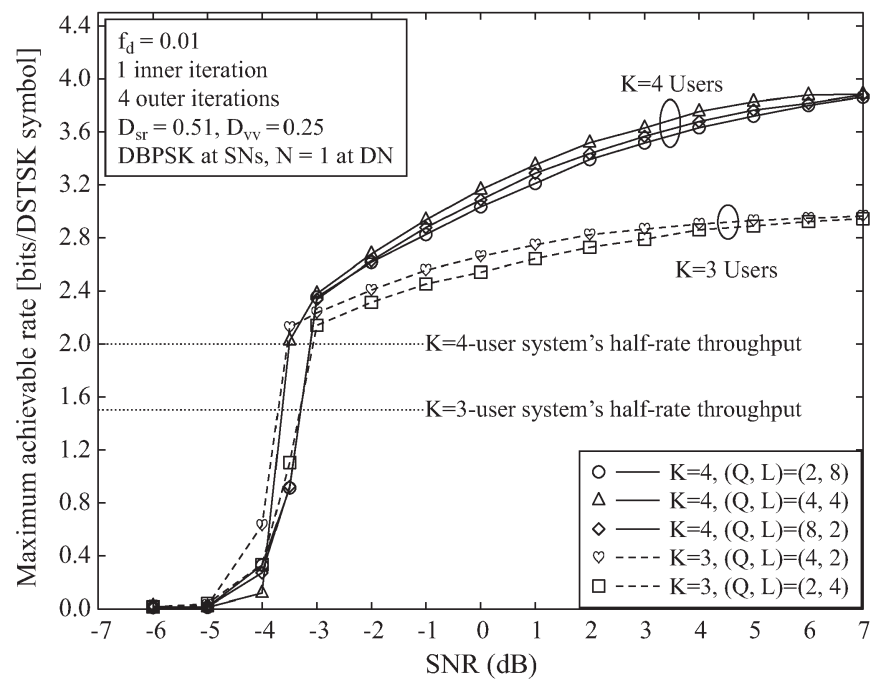

Fig. 4. Maximum achievable rates of different combinations $(Q, \mathcal{L})$ for supporting $K=3$ and 4 users, associated with DBPSK at SNs and $N=1$ receive antenna at $\mathrm{DN}$.

a few decibels beyond the system's capacity, as exemplified latter by the BER simulation results of Fig. 7, and we may focus our attention on the system's capacity point and its immediate vicinity. It can be seen that the combination of $(Q, \mathcal{L})=(4,2)$ reach the half-rate throughput of $1.5 \mathrm{bits} / \mathrm{symbol}$ at $\mathrm{SNR}=$ $-3.8 \mathrm{~dB}$, whereas the combination of $(Q, \mathcal{L})=(2,4)$ reach the half-rate throughput at $\mathrm{SNR}=-3.4 \mathrm{~dB}$. Beyond this point, the combination $(Q, \mathcal{L})=(4,2)$ still outperforms the other combination $(Q, \mathcal{L})=(2,4)$. Clearly, the combination $(Q, \mathcal{L})=$ $(4,2)$ is the best configuration based on the system's maximum achievable rate. This agrees with the results produced by the proposed MMBCS algorithm as listed in Table III, which also confirms that $\left(Q_{\text {opt }}, \mathcal{L}_{\text {opt }}\right)=(4,2)$ is the best configuration to support $K=3$ users.

The three maximum achievable rates for the system with $K=4$ users are shown in Fig. 4 as solid curves, where we observe that the combination $(Q, \mathcal{L})=(4,4)$ reaches the half-rate throughput point at approximately $\mathrm{SNR}=-3.7 \mathrm{~dB}$, whereas the other two combinations require about $\mathrm{SNR}=-3 \mathrm{~dB}$. Additionally, beyond its half-rate SNR point, the combination $(Q, \mathcal{L})=(4,4)$ outperforms the other two combinations, in terms of the maximum achievable rate. Therefore, $(Q, \mathcal{L})=$ $(4,4)$ can be regarded as the optimal combination, which again agrees with the results of Table III, provided by the proposed MMBCS algorithm. It is worth pointing out that all the legitimate DSTSK configurations $(Q, \mathcal{L})$ associated with the same number of users $K$ have the same system complexity. According to the operational principle of our SISO-MSDSD invoked for DSTSK, the size of the search set is determined by the product $Q \cdot \mathcal{L}$, which in fact depends on the number of users $K$. Note that all the combinations have the same product $Q \cdot \mathcal{L}$ as $\log _{2}(Q \cdot \mathcal{L})=K$. In other words, given the number of users $K$, the lower bounded complexity of our SISO-MSDSDaided cooperative system is determined. This lower bounded complexity is given in Table I as $N_{c}$, where the complexity is quantified in terms of the number of complex additions, multiplications, and absolute value calculations per user. 


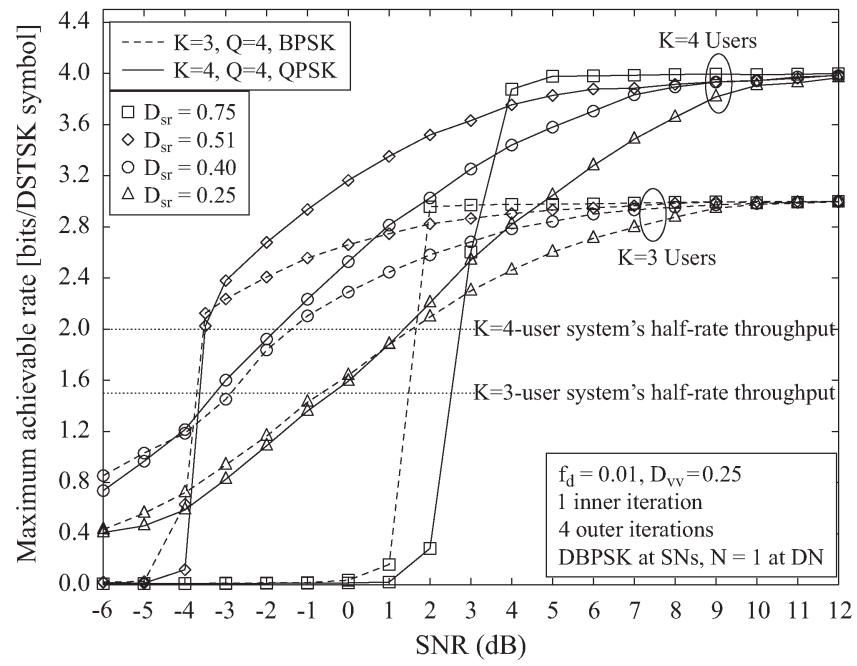

Fig. 5. Effects of different SR distances on the system's maximum achievable rate for the systems $\left(Q_{\mathrm{opt}}, \mathcal{L}_{\mathrm{opt}}\right)=(4,2)$ and $\left(Q_{\mathrm{opt}}, \mathcal{L}_{\mathrm{opt}}\right)=(4,4)$ to support $K=3$ and $K=4$ users, respectively.

Fig. 5 depicts the effects of the different SR distances $D_{s r}$ on the maximum achievable rate for the DSTSK systems $\left(Q_{\text {opt }}, \mathcal{L}_{\text {opt }}\right)=(4,2)$ to support $K=3$ users and $\left(Q_{\text {opt }}\right.$, $\left.\mathcal{L}_{\text {opt }}\right)=(4,4)$ to support $K=4$ users, respectively, where the SR distances are chosen as $D_{s r} \in\{0.25,0.40,0.51,0.75\}$. The maximum achievable rates associated with the system of $K=3$ users are shown as dashed curves, where it can be seen that, although the curve of $D_{s r}=0.51$ exhibits a lower throughput in the low-SNR region, it reaches the half-rate throughput point at $\mathrm{SNR}=-3.8 \mathrm{~dB}$, which is smaller than the SNR values required by the other three SR distances. Additionally, the $D_{s r}=0.51$ scenario outperforms the others beyond its half-rate SNR point and up to about $\mathrm{SNR}=2 \mathrm{~dB}$, where a vanishingly low BER is achieved, as shown in Fig. 7. The four solid curves in Fig. 5 correspond to the maximum achievable rates associated with the four SR distances $D_{s r}$ for the system of $K=4$ users. It can be seen that, similar to the three-user system, in the low-SNR region, the curve of the $D_{s r}=0.51$ case for the four-user system is relatively low, but it reaches the half-rate point at $\mathrm{SNR}=-3.7 \mathrm{~dB}$, which is earlier than the other three SR distance cases. Furthermore, the $D_{s r}=0.51$ scenario outperforms the other three cases up to $\mathrm{SNR}=3 \mathrm{~dB}$. Therefore, we can conclude that the SR distance $D_{s r}=0.51$ is optimal for this system configuration to support both $K=$ 3 and 4 users, in terms of the system maximum achievable rate.

Fig. 6 portrays the EXIT chart [35] of the DSTSK-aided MUSRC system $\left(Q_{\text {opt }}, \mathcal{L}_{\text {opt }}\right)=(4,4)$ supporting $K=4$ users and relying on a detection window size of $N_{w}=4$. It can be seen that an open tunnel exists between the EXIT curves of the inner MSDSD-URC decoder and of the outer RSC decoder at $\mathrm{SNR}=-0.8 \mathrm{~dB}$. The Monte-Carlo-simulation-based staircase-shaped decoding trajectory, which closely matched the EXIT curves, is also provided at SNR $=-0.8 \mathrm{~dB}$. This trajectory shows that the point of perfect convergence at $(1.0,1.0)$ is reached with the aid of $I_{\text {outer }}=4$ iterations, implying that our system supporting $K=4$ users and relying on an MSDSD

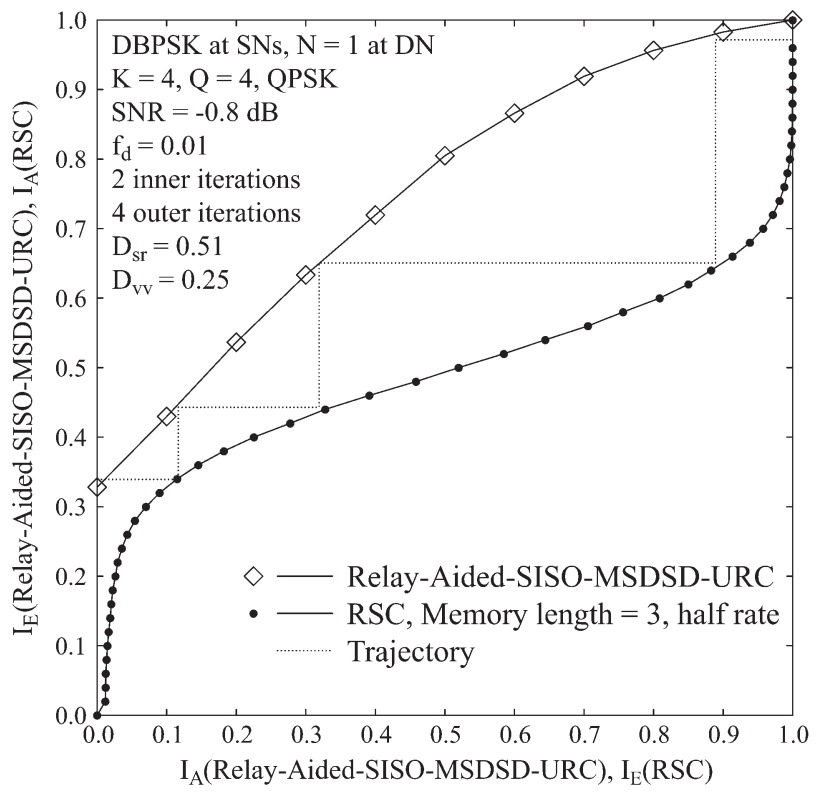

Fig. 6. EXIT characteristics and the corresponding decoding trajectory of the proposed cooperative system $\left(Q_{\text {opt }}, \mathcal{L}_{\text {opt }}\right)=(4,4)$ for the given number of $K=4$ users at SNR $=-0.8 \mathrm{~dB}$.

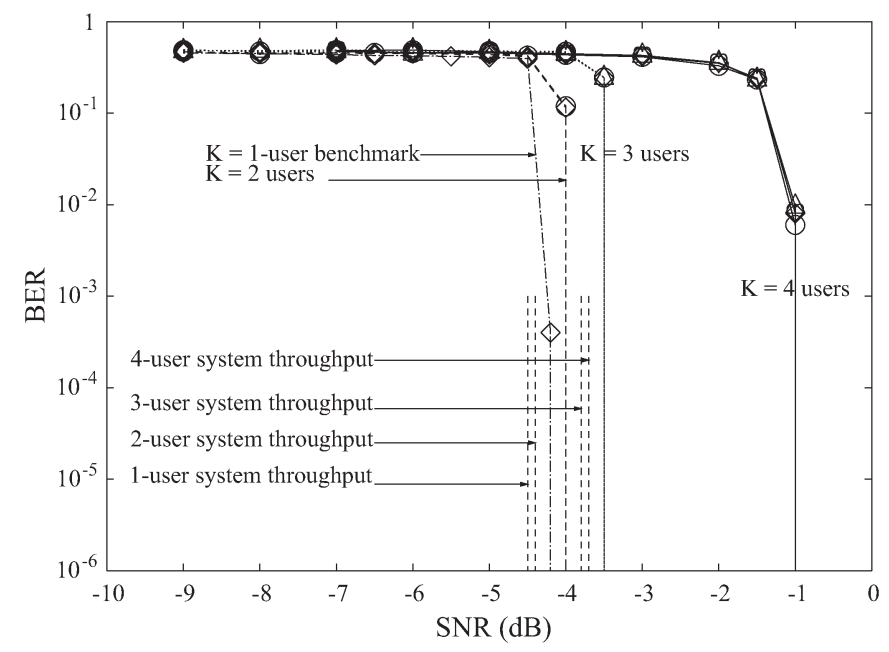

Fig. 7. BER performance of the proposed cooperative system supporting $K=1,2,3$, and 4 users and the corresponding system capacity. The system employs DBPSK at SNs and $N=1$ receive antenna at the DN.

detection window size of $N_{w}=4$ is capable of achieving a vanishingly low error probability beyond the point of SNR = $-0.8 \mathrm{~dB}$, as is confirmed by the BER curves of the $K=4$-user system characterized in Fig. 7.

In a multiuser system exhibiting fairness, the quality of service $(\mathrm{Q} o \mathrm{~S})$ should be identical for each user. We plot the BER performance of the individual users in Fig. 7 for the cases of $K=2,3$, and 4 . The BER curve group indicated by " $K=4$ users" contains the four individual users' BERs, whereas the BER curve group of " $K=3$ users" represents the three individual users' BERs. Finally, the two BER curves of the two-user system and of the single-user benchmark are grouped under the labels of " $K=2$ users" and " $K=1$-user benchmark", respectively. It is shown in Fig. 7 that, for the 


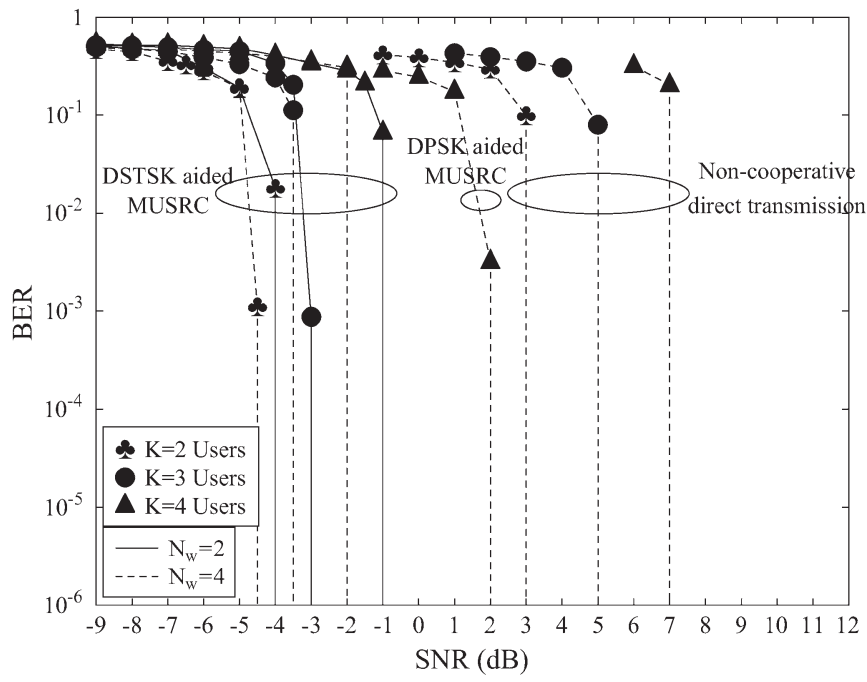

Fig. 8. Effects of MSDSD window size $N_{w}$ on the BER performance of the proposed DSTSK-aided MUSRC system, which employs DBPSK at SNs and $N=1$ receive antenna at the $\mathrm{DN}$, and the BER performance comparison with the DSTSK noncooperative direct transmission system and the conventional DPSK-aided MUSRC system.

system supporting $K \in\{2,3,4\}$ users, all the users in each case share the same BER performance, implying that the same QoS is guaranteed for each of the $K$ users. Additionally, the system throughput for each of the $K=1,2,3$, and 4 scenarios are also plotted in Fig. 7, where it can be seen that the differences between the capacity lines and "Turbo cliffs" are usually less than $3 \mathrm{~dB}$, implying that our proposed DSTSK-aided MUSRC scheme is indeed capable of achieving near-capacity performance with the aid of three-stage serialconcatenated coding scheme.

Fig. 8 shows the effects of the MSDSD detection window size $N_{w}$ on the attainable BER performance of our proposed system supporting $K \in\{2,3,4\}$ users. It can be seen from the results shown in Fig. 8 that the BER performance is improved as the window size increases from $N_{w}=2$ to 4 , at the cost of higher complexity. However, the complexity of the proposed MSDSD scheme does not increase exponentially; hence, the system's complexity remains acceptable for a detection window size of $N_{w}=4$. The BER performances of noncooperative three-stage serial-concatenated turbo-codingaided DSTSK schemes relying on an MSDSD window size of $N_{w}=4$ are also included in Fig. 8 for comparison with our proposed DSTSK-aided MUSRC system. Observe in Fig. 8 that the proposed DSTSK-aided MUSRC system significantly outperforms the noncooperative DSTSK scheme. We also simulated a DPSK-aided MUSRC system, where the DSTSK scheme based on $N_{r}=2$ RNs per VAA invoked for relaying in our proposed DSTSK-aided MUSRC was replaced by the conventional DPSK-based relaying relying on a single $\mathrm{RN}$ per VAA. The performance of this DPSK-aided MUSRC system supporting $K=4$ users and with an MSDSD window size of $N_{w}=4$ is also included in Fig. 8. Observe in Fig. 8 that the proposed DSTSK-aided MUSRC offers a performance gain of about $3 \mathrm{~dB}$ over this DPSK-aided MUSRC, which is achieved at the cost of imposing slightly higher complexity than the DPSK-aided MUSRC system, as confirmed by the complexity bounds $N_{c}$ of the two MUSRC systems given in Table IV.

\section{B. DQPSK for SR Transmission and DSTSK $(2,2,2, Q, \mathcal{L})$ for RD Transmission}

We also considered the proposed DSTSK-aided MUSRC system employing DQPSK for the SR transmission and a DSTSK scheme of $\operatorname{DSTSK}(2,2,2, Q, \mathcal{L})$ for the RD transmission, associated with $N_{r}=2$ relays in each of the two VAA groups and a DN equipped with $N=2$ antennas. We first quantified the maximum achievable rates for all the legitimate DSTSK configurations $(Q, \mathcal{L})$ supporting $K \in\{3,4\}$ users, respectively, in conjunction with an MSDSD window size of $N_{w}=4$. The SR distance was chosen as $D_{s r}=0.4$, which will be shown to be the optimal distance for this DSTSKaided MUSRC system. The simulation results obtained are shown in Fig. 9, where the maximum achievable rates of the two DSTSK configurations supporting $K=3$ users are depicted as dashed cures, whereas the maximum achievable rates of the three DSTSK configurations supporting $K=4$ users are shown as solid curves. The results in Fig. 9 demonstrate that configuration $(Q, \mathcal{L})=(4,2)$ is optimal for the three-user system, whereas the configuration $(Q, \mathcal{L})=(4,4)$ is optimal for the four-user system, in terms of the maximum achievable rate. This observation agrees with the results provided by the proposed MMBCS algorithm listed in Table III, which again demonstrates the power of the MMBCS algorithm in selecting the optimal DSTSK configuration $\left(Q_{\text {opt }}, \mathcal{L}_{\text {opt }}\right)$ for the given number of users $K$.

The effects of SR distances $D_{s r}$ on the maximum achievable rate of the proposed cooperative system for the optimal combinations of $\left(Q_{\mathrm{opt}}, \mathcal{L}_{\mathrm{opt}}\right)=(4,2)$ and $\left(Q_{\mathrm{opt}}, \mathcal{L}_{\mathrm{opt}}\right)=(4,4)$ supporting $K \in\{3,4\}$ users, respectively, are portrayed in Fig. 10, where the SR distances chosen for our simulation were $D_{s r} \in\{0.2,0.3,0.4,0.50\}$. The four dashed curves in Fig. 10 depict the maximum achievable rates for the $K=3$-user scenario, where it can be seen clearly that the $D_{s r}=0.4$ scenario outperforms the other three distances. The four maximum achievable rates for the $K=4$-user system are shown in Fig. 10 as solid curves, and it is clearly that the case of $D_{s r}=0.4$ outperforms the other three cases. Hence, for the DSTSKaided MUSRC system employing the DQPSK for the SR transmission and the DSTSK scheme of $\operatorname{DSTSK}(2,2,2, Q, \mathcal{L})$ for the $\mathrm{RD}$ transmission, the $\mathrm{SR}$ distance of $D_{s r}=0.4$ is optimal.

Fig. 11 portrays the EXIT chart for the DSTSK-aided MUSRC system $\left(Q_{\text {opt }}, \mathcal{L}_{\text {opt }}\right)=(4,4)$ supporting $K=4$ users and relying on a detection window size of $N_{w}=4$. It can be seen that an open tunnel exists at SNR $=-2.4 \mathrm{~dB}$. The MonteCarlo-simulation-based stair-case-shaped decoding trajectory shows that the point of perfect convergence at $(1.0,1.0)$ is reached with the aid of $I_{\text {outer }}=4$ iterations. Hence, our system supporting $K=4$ users with an MSDSD detection window size of $N_{w}=4$ is capable of achieving a vanishingly low error probability beyond the point of $\mathrm{SNR}=-2.4 \mathrm{~dB}$, which is also confirmed by the BER curves of the $K=4$-users system 
TABLE IV

COMPARISON OF THE COMPLEXITy BOUNDS $N_{c}$ [28] FOR THE DSTSK-AIDED MUSRC SYSTEM AND THE CONVENTIONAL DPSK-AIDED MUSRC System, Both ASSOciated With $N_{w}=4$, DBPSK at SNS AND $N=1$ Receive Antenna AT the DN

\begin{tabular}{|c|c|c|c|c|}
\hline Users & $K=2$ & $K=3$ & $K=4$ & $K=5$ \\
\hline DSTSK aided MUSRC $\left(N_{r}=2\right.$ per VAA $)$ & $N_{c}=180$ & $N_{c}=200$ & $N_{c}=250$ & $N_{c}=360$ \\
\hline DPSK aided MUSRC $\left(N_{r}=1\right.$ per VAA $)$ & $N_{c}=35$ & $N_{c}=46$ & $N_{c}=70$ & $N_{c}=150$ \\
\hline
\end{tabular}

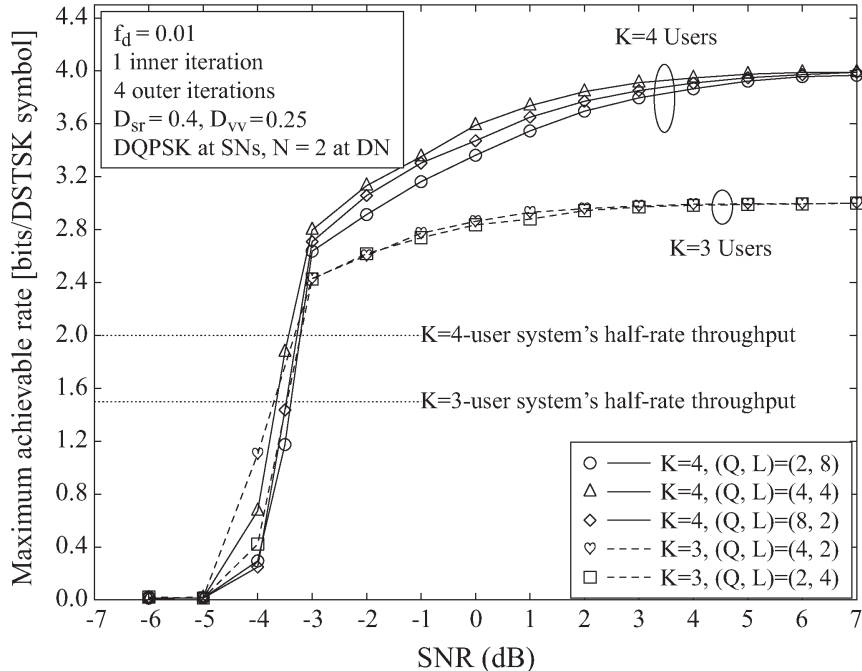

Fig. 9. Maximum achievable rates of different combinations $(Q, \mathcal{L})$ for supporting $K=3$ and 4 users, associated with DQPSK at SNs and $N=2$ receive antennas at $\mathrm{DN}$.

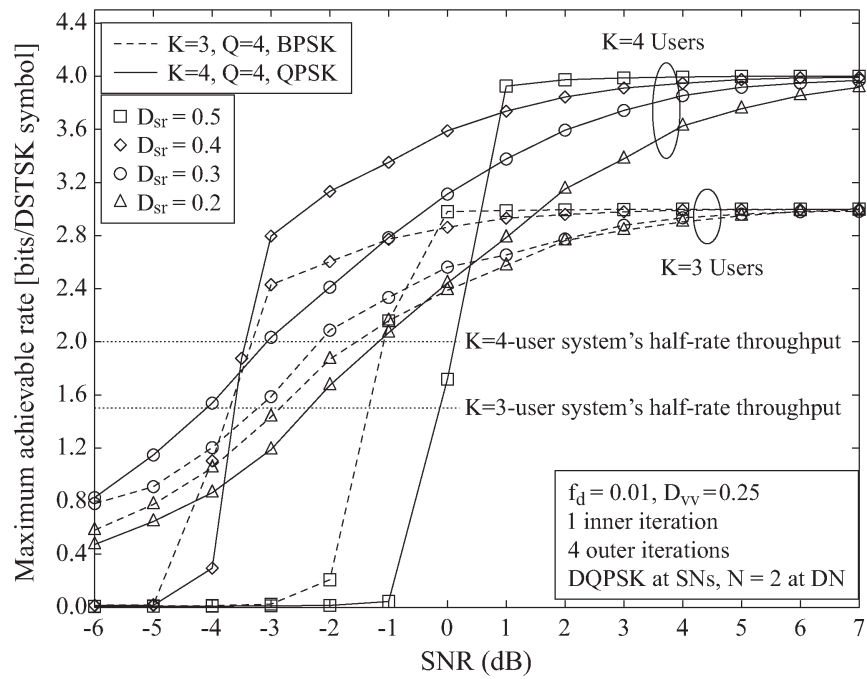

Fig. 10. Effects of different SR distances on the system's maximum achievable rate for the systems $\left(Q_{\mathrm{opt}}, \mathcal{L}_{\mathrm{opt}}\right)=(4,2)$ and $\left(Q_{\mathrm{opt}}, \mathcal{L}_{\mathrm{opt}}\right)=(4,4)$ to support $K=3$ and $K=4$ users, respectively.

provided in Fig. 12. In Fig. 12, we plot the BER performance of the individual users for the systems supporting $K=2,3$, and 4 users, respectively, where it can be seen clearly that all the users in each system exhibit an identical BER performance, indicating that the same QoS is guaranteed for each of the $K$ users. The $K=1$ user performance is included as a benchmark. By comparing the BER "Turbo cliffs" with their corresponding capacity lines in Fig. 12, we observe that our DSTSK-aided MUSRC scheme is capable of achieving near-capacity perfor-

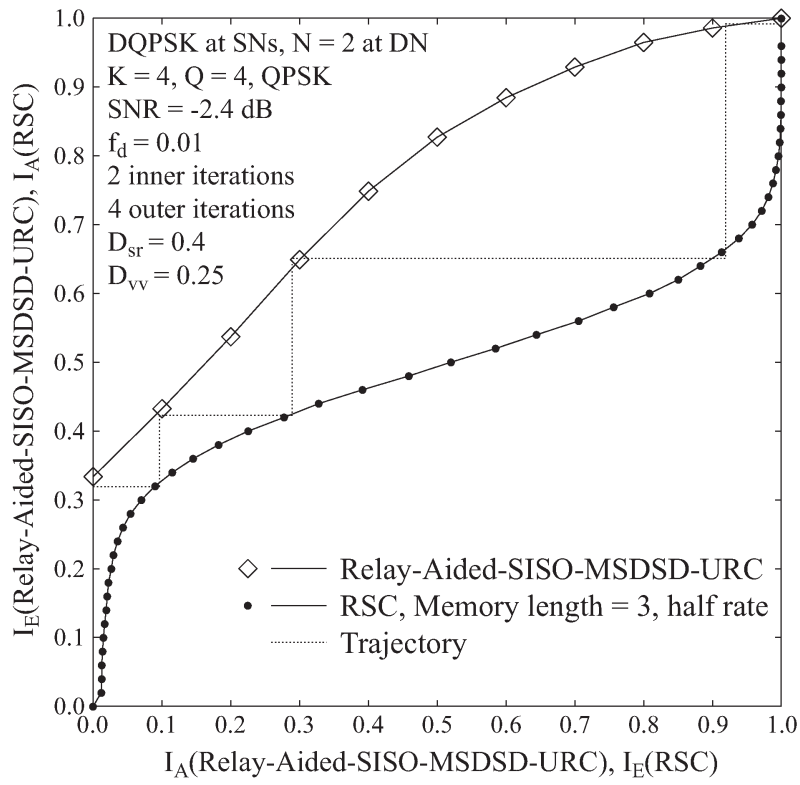

Fig. 11. EXIT characteristics and the corresponding decoding trajectory of the proposed cooperative system $\left(Q_{\mathrm{opt}}, \mathcal{L}_{\mathrm{opt}}\right)=(4,4)$ for the given number of $K=4$ users at SNR $=-2.4 \mathrm{~dB}$.

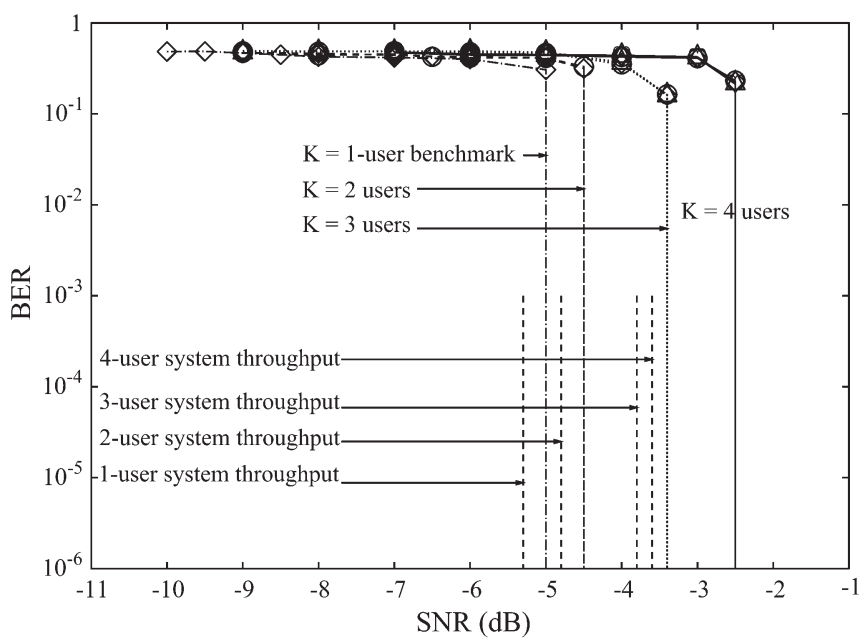

Fig. 12. BER performance of the proposed cooperative system supporting $K=1,2,3$ and 4 users and the corresponding system capacity. The system employs DQPSK at SNs and $N=2$ receive antennas at the DN.

mance with the aid of three-stage serial-concatenated coding scheme.

\section{CONClusion}

We have proposed a DSTSK-aided MUSRC system, which is capable of supporting multiple users by exploiting the flexibility of the DSTSK MIMO scheme relying on VAA-based 
cooperative relaying. By adopting the two-phase successiverelaying architecture, the conventional 50\% throughput loss has been recovered at the cost of supporting fewer users. We have also proposed the MMBCS algorithm for selecting the most appropriate or optimal DSTSK configuration for supporting different number of users, and the effectiveness of this optimal DSTSK configuration selection algorithm has been verified by the maximum-achievable-rate-based simulation results. Additionally, three-stage MSDSD-URC-RSC turbo encoding/decoding has been conceived for multiuser DSTSK-based cooperative relaying, and the relevant simulation results have shown that the proposed system is capable of achieving a near-capacity performance, while maintaining low complexity.

\section{REFERENCES}

[1] N. Chiurtu, B. Rimoldi, and E. Telatar, "On the capacity of multi-antenna Gaussian channels," in Proc. IEEE Int. Symp. Inf. Theory, Washington, DC, USA, Jun. 24-29, 2001, p. 53.

[2] K. C. Huang and Z. Wang, "Millimeter-wave circular polarized beamsteering antenna array for gigabit wireless communications," IEEE Trans. Antennas Propag., vol. 54, no. 2, pp. 743-746, Feb. 2006.

[3] R. Y. Mesleh, H. Haas, S. Sinanovic, C. W. Ahn, and S. Yun, "Spatial modulation," IEEE Trans. Veh. Technol., vol. 57, no. 4, pp. 2228-2241, Jul. 2008.

[4] M. D. Renzo, H. Haas, and P. M. Grant, "Spatial modulation for multipleantenna wireless systems: A survey," IEEE Commun. Mag., vol. 49, no. 12, pp. 182-191, Dec. 2011.

[5] J. Jeganathan, A. Ghrayeb, L. Szczecinski, and A. Ceron, "Space shift keying modulation for MIMO channels," IEEE Trans. Wireless Commun., vol. 8, no. 7, pp. 3692-3703, Jul. 2009.

[6] S. Sugiura, S. Chen, and L. Hanzo, "A universal space-time architecture for multiple-antenna aided systems," IEEE Commun. Surveys Tuts., vol. 14, no. 2, pp. 401-420, 2nd Quart., 2012.

[7] Y. Fan, C. Wang, H. V. Poor, and J. S. Thompson, "Cooperative multiplexing: Toward higher spectral efficiency in multiple-antenna relay networks," IEEE Trans. Inf. Theory, vol. 55, no. 9, pp. 3909-3926, Sep. 2009.

[8] P. Zhang, J. Yuan, J. Chen, J. Wang, and J. Yang, "Analyzing amplifyand-forward and decode-and-forward cooperative strategies in Wyner's channel model," in Proc. IEEE WCNC, Budapest, Hungary, Apr. 5-8, 2009, pp. 1-5.

[9] N. Serafimovski, S. Sinanovic, M. Di Renzo, and H. Haas, "Dual-hop Spatial Modulation (Dh-SM)," in Proc. IEEE VTC-Spring, Budapest, Hungary, May 15-18, 2011, pp. 1-5.

[10] D. Yang, C. Xu, L.-L. Yang, and L. Hanzo, "Transmit-diversity-assisted space-shift keying for colocated and distributed/cooperative MIMO elements," IEEE Trans. Veh. Technol., vol. 60, no. 6, pp. 2864-2869, Jul. 2011.

[11] M. Dohler, A. Gkelias, and H. Aghvami, "A resource allocation strategy for distributed MIMO multi-hop communication systems," IEEE Commun. Lett., vol. 8, no. 2, pp. 99-101, Feb. 2004.

[12] H. J. An and H. K. Song, "Cooperative communication in SIMO systems with multiuser-OFDM," IEEE Trans. Consum. Electron., vol. 53, no. 2, pp. 339-343, May 2007.

[13] C. Xu, E. Ternon, S. Sugiura, S. X. Ng, and L. Hanzo, "Multiple-symbol differential sphere decoding aided cooperative differential space-time spreading for the asynchronous CDMA uplink," in Proc. IEEE GLOBECOM, Houston, TX, USA, Dec. 5-9, 2011, pp. 1-5.

[14] C. Kotchasarn, "Cooperative diversity for synchronous uplink DS-CDMA transmission over Rayleigh frequency flat fading channel," in Proc. IEEE SCOReD, UPM Serdang, Malaysia, Nov. 16-18, 2009, pp. 53-56.

[15] W. Fang, L. L. Yang, and L. Hanzo, "Single-user performance of uplink DS-CDMA using relay-assisted diversity," in Proc. 17th IEEE Int. Symp. PIMRC, Helsinki, Finland, Sep. 11-14, 2006, pp. 1-5.

[16] Y. Yang, "Information-guided relay selection for high throughput in halfduplex relay channels," in Proc. IEEE GLOBECOM, Honolulu, HI, USA, Nov. 30-Dec. 4, 2009, pp. 1-5.

[17] Y. Yang and S. Aissa, "Information-guided transmission in decode-andforward relaying systems: Spatial exploitation and throughput enhance- ment," IEEE Trans. Wireless Commun., vol. 10, no. 7, pp. 2341-2351, Jul. 2011.

[18] Y. Fan, C. Wang, J. Thompson, and H. V. Poor, "Recovering multiplexing loss through successive relaying using repetition coding," IEEE Trans. Wireless Commun., vol. 6, no. 12, pp. 4484-4493, Dec. 2007.

[19] M. Di Renzo and H. Haas, "Space Shift Keying (SSK) modulation with partial channel state information: Optimal detector and performance analysis over fading channels," IEEE Trans. Commun., vol. 58, no. 11, pp. 3196-3210, Nov. 2010

[20] E. Basar, U. Aygolu, E. Panayirci, and H. V. Poor, "Performance of spatial modulation in the presence of channel estimation errors," IEEE Commun. Lett., vol. 16, no. 2, pp. 176-179, Feb. 2012.

[21] S. S. Ikki and R. Mesleh, "A general framework for performance analysis of Space Shift Keying (SSK) modulation in the presence of Gaussian imperfect estimations," IEEE Commun. Lett., vol. 16, no. 2, pp. 228-230, Feb. 2012.

[22] M. Di Renzo, D. D. Leonardis, F. Graziosi, and H. Haas, "Space Shift Keying (SSK-) MIMO with practical channel estimates," IEEE Trans. Commun., vol. 60, no. 4, pp. 998-1012, Apr. 2012.

[23] C. Shin, R. W. Heath, and E. J. Powers, "Blind channel estimation for MIMO-OFDM systems," IEEE Trans. Veh. Technol., vol. 56, no. 2, pp. 670-685, Mar. 2007.

[24] L. Tang, R. W. Liu, V. C. Soon, and Y. F. Huang, "Indeterminacy and identifiability of blind identification," IEEE Trans. Circuits Syst., vol. 38, no. 5, pp. 499-509, May 1991.

[25] S. Sugiura, S. Chen, H. Haas, P. M. Grant, and L. Hanzo, "Coherent versus non-coherent decode-and-forward relaying aided cooperative space-time shift keying," IEEE Trans. Commun., vol. 59, no. 6, pp. 1707-1719, Jun. 2011.

[26] L. Wang and L. Hanzo, "The amplify-and-forward cooperative uplink using multiple-symbol differential sphere-detection," IEEE Signal Process. Lett., vol. 16, no. 10, pp. 913-916, Oct. 2009.

[27] D. Divsalar and M. K. Simon, "Multiple-symbol differential detection of MPSK," IEEE Trans. Commun., vol. 38, no. 3, pp. 300-308, Mar. 1990.

[28] L. Lampe, R. Schober, V. Pauli, and C. Windpassinger, "Multiple-symbol differential sphere decoding," IEEE Trans. Commun., vol. 53, no. 12, pp. 1981-1985, Dec. 2005.

[29] V. Pauli, L. Lampe, and R. Schober, “"Turbo DPSK' using soft multiplesymbol differential sphere decoding," IEEE Trans. Inf. Theory, vol. 52, no. 4, pp. 1385-1398, Apr. 2006.

[30] L. Hanzo, L. L. Yang, E. L. Kuan, and K. Yen, Single- and Multi-Carrier DS-CDMA: Multi-User Detection, Space-Time Spreading, Synchronisation and Standards. Chichester, U.K.: Wiley, 2003.

[31] L. L. Yang and W. Fang, "Performance of cellular DS-CDMA systems using distributed antennas," in Proc. 17th IEEE Int. Symp. PIMRC, Helsinki, Finland, Sep. 11-14, 2006, pp. 1-5.

[32] L. Hanzo, M. Münster, B.-J. Choi, and T. Keller, OFDM and MC-CDMA for Broadband Multi-User Communications, WLANs and Broadcasting. Chichester, U.K.: Wiley, 2003.

[33] L. Hanzo, J. S. Blogh, and S. Ni, 3G, HSPA and FDD versus TDD Networking: Smart Antennas and Adaptive Modulation. Chichester, U.K.: Wiley, 2008.

[34] S. Sugiura, S. Chen, and L. Hanzo, "Coherent and differential spacetime shift keying: A dispersion matrix approach," IEEE Trans. Commun., vol. 58, no. 11, pp. 3219-3230, Nov. 2010.

[35] L. Hanzo, O. R. Alamri, M. El-Hajjar, and N. Wu, Near-Capacity MultiFunctional MIMO Systems: Sphere-Packing, Iterative Detection and Cooperation. Chichester, U.K.: Wiley, 2009.

[36] Z. Pi and F. Khan, "An introduction to millimeter-wave mobile broadband systems," IEEE Commun. Mag, vol. 49, no. 6, pp. 101-107, Jun. 2011.

[37] T. Kamalakis, I. Neokosmidis, A. Tsipouras, S. Pantazis, and I. Andrikopoulos, "Hybrid free space optical/millimeter wave outdoor links for broadband wireless access networks," in Proc. 18th IEEE Int. Symp. PIMRC, Athens, Greece, Sep. 3-7, 2007, pp. 1-5.

[38] C. Xu, S. X. Ng, and L. Hanzo, "Near-capacity irregular convolutional coded cooperative differential linear dispersion codes using multiplesymbol differential detection," IEEE Signal Process. Lett., vol. 18, no. 3, pp. 173-176, Mar. 2011.

[39] X. Chao, S. Sugiura, S. X. Ng, and L. Hanzo, "Reduced-complexity noncoherently detected differential space-time shift keying," IEEE Signal Process. Lett., vol. 18, no. 3, pp. 153-156, Mar. 2011.

[40] R. W. Heath, Jr. and A. J. Paulraj, "Linear dispersion codes for MIMO systems based on frame theory," IEEE Trans. Signal Process., vol. 50, no. 10, pp. 2429-2441, Oct. 2002. 


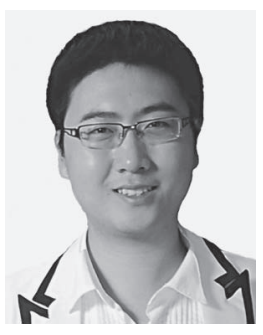

Peichang Zhang received the B.Eng. degree (with first-class honors) in electronic engineering from the University of Central Lancashire, Lancashire, U.K., in 2009 and the M.Sc. degree with distinction in wireless communications from the University of Southampton, Southampton, U.K., in 2010. He is currently working toward the Ph.D. degree with the Communications, Signal Processing, and Control Research Group, Department of Electronics and Computer Science, University of Southampton.

His research interests include cooperative communications, coherent and noncoherent detection, iterative detection, and channel estimation.

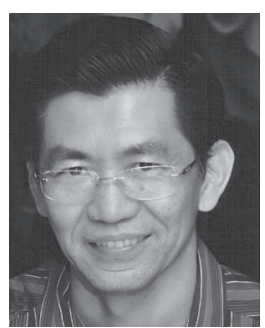

Sheng Chen (M'90-SM'97-F'08) received the B.Eng. degree in control engineering from the East China Petroleum Institute, Dongying, China, in 1982, the Ph.D. degree in control engineering from City University London, London, U.K., in 1986, and the D.Sc. degree from the University of Southampton, Southampton, U.K, in 2005.

From 1986 to 1999, He held research and academic appointments with the University of Sheffield, South Yorkshire, the University of Edinburgh, and the University of Portsmouth, all in the U.K. Since 1999, he has been with Electronics and Computer Science, the University of Southampton, where he is currently a Professor of intelligent systems and signal processing. He is also a Distinguished Adjunct Professor with King Abdulaziz University, Jeddah, Saudi Arabia. He is an Institute for Scientific Information highly cited researcher in the engineering category (March 2004). He is the author of over 460 research papers. His recent research interests include adaptive signal processing, wireless communications, modeling and identification of nonlinear systems, neural network and machine learning, intelligent control system design, evolutionary computation methods, and optimization.

Dr. Chen is a Fellow of The Institution of Engineering and Technology and a Chartered Engineer.

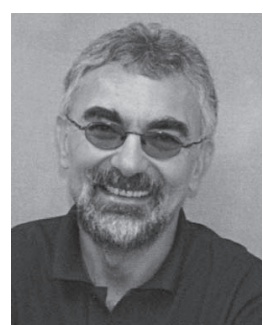

Lajos Hanzo (M'91-SM'92-F'04) received the M.S. degree (with first-class honors) in electronics and the Ph.D. degree from the Technical University of Budapest, Budapest, Hungary, in 1976 and 1983 , respectively, the D.Sc. degree from the University of Southampton, Southampton, U.K., in 2004, and the "Doctor Honoris Causa" degree from the Technical University of Budapest in 2009.

During his 35-year career in telecommunications, he has held various research and academic posts in Hungary, Germany, and the U.K. Since 1986, he has been with the School of Electronics and Computer Science, University of Southampton, where he holds the Chair in Telecommunications. Since 2009, he has been a Chaired Professor with Tsinghua University, Beijing, China. $\mathrm{He}$ is currently directing a 100 -strong academic research team, working on a range of research projects in the field of wireless multimedia communications sponsored by industry; the Engineering and Physical Sciences Research Council, U.K.; the European Information Society Technologies Program; and the Mobile Virtual Centre of Excellence, U.K. He is an enthusiastic supporter of industrial and academic liaison and offers a range of industrial courses. $\mathrm{He}$ has successfully supervised $80 \mathrm{Ph}$.D. students, coauthored 20 JohnWiley/IEEE Press books on mobile radio communications totaling in excess of 10000 pages, published more than 1250 research entries on IEEE Xplore, and presented keynote lectures. For further information on research in progress and associated publications, see http://www-mobile.ecs.soton.ac.uk/.

Dr. Hanzo is a Fellow of the Royal Academy of Engineering, U.K., a Fellow of the Institution of Electrical Engineers, and a Governor of the IEEE Vehicular Technology Society. He has been a Technical Program Committee Chair and a General Chair for IEEE conferences. During 2008-2012, he was the Editor-inChief of the IEEE Press. He has received a number of distinctions. 